\title{
Transformation of the multipolar components of gravitational radiation under rotations and boosts
}

\author{
Leonardo Gualtieri ${ }^{1 *}$, Emanuele Berti ${ }^{2 \dagger}$, Vitor Cardoso ${ }^{3,4 \ddagger}$ Ulrich Sperhake $^{5 \S}$ \\ 1 Dipartimento di Fisica, Università di Roma "Sapienza" and Sezione INFN Roma1, P.A. Moro 5, 00185, Roma, Italy \\ 2 Jet Propulsion Laboratory, California Institute of Technology, Pasadena, CA 91109, USA \\ 3 Centro Multidisciplinar de Astrofísica - CENTRA, Dept. de Física, \\ Instituto Superior Técnico, Av. Rovisco Pais 1, 1049-001 Lisboa, Portugal \\ 4 Department of Physics and Astronomy, The University of Mississippi, University, MS 38677-1848, USA and \\ 5 Theoretisch Physikalisches Institut, Friedrich Schiller Universität, 07743 Jena, Germany
}

\begin{abstract}
We study the transformation of multipolar decompositions of gravitational radiation under rotations and boosts. Rotations to the remnant black hole's frame simplify the waveforms from the merger of generic spinning black hole binaries. Boosts may be important to get an accurate gravitational-wave phasing, especially for configurations leading to large recoil velocities of the remnant. As a test of the formalism we revisit the classic problem of point particles falling into a Schwarzschild black hole. Then we highlight by specific examples the importance of choosing the right frame in numerical simulations of unequal-mass, spinning binary black-hole mergers.

PACS numbers: 04.30.-w, 04.25.D-, 04.25.dg, 04.70.Bw, 02.20.-a
\end{abstract}

\section{INTRODUCTION}

Multipolar expansions are a fundamental tool in the study of gravitational radiation. Such expansions can be performed using different sets of basis functions (see [1] for a review). It is common practice in numerical relativity simulations to decompose the Weyl scalar $\Psi_{4}$ (a quantity related to outgoing gravitational radiation) as a sum over the Newman-Penrose spin-weighted spherical harmonics (SWSHs) of spin-weight $s$, commonly denoted by ${ }_{s} Y_{l m}$. SWSHs reduce to ordinary spherical harmonics for $s=0$, and $|s|=2$ is the spin-weight of interest for gravitational radiation [2, 3].

Compact binaries are one of the most promising candidate sources of detectable gravitational waves for Earthbased and space-based interferometers. The post-Newtonian approximation, an analytical expansion of Einstein's equations valid for orbital velocities $V / c \ll 1$, is usually employed to describe the early inspiral of compact binaries. The post-Newtonian expansion becomes increasingly inaccurate late in the inspiral, when orbital velocities become large. At these late stages, time evolutions of compact binaries in numerical relativity are necessary. High-accuracy numerical evolutions are difficult for various reasons, including the approximate nature of the initial data, errors arising from discretization and boundary effects. Post-Newtonian expansions and numerical calculations must be cross-validated by systematic comparisons of the resulting waveforms, in order to determine a common region of validity and to build reliable phenomenological templates [4, 5, 6, 7, 8, 9, 10, 11, 12. Since numerical waveforms are usually decomposed in SWSHs, significant efforts have recently been devoted to study multipolar SWSH expansions both within post-Newtonian theory [13, 14] and in connection with numerical simulations [15, 16, 17, 18, 19].

This paper deals with the transformation properties of SWSH decompositions of gravitational radiation under rotations and boosts. We will mostly focus on the radiation emitted during the inspiral, merger and ringdown of compact binaries, but our main results are general enough that they should be useful in more general situations.

The transformation properties of gravitational waves have been studied as part of the general problem of spacetime transformations in the sixties 22, 20, 21, 22]. It was realized that the asymptotic symmetries of asymptotically flat spacetimes form an infinite-dimensional group, now known as the Bondi-Metzner-Sachs (BMS) group, and that Lorentz transformations are a subgroup of the BMS group. Later on, the transformations of SWSHs under the BMS group have been investigated in a classic paper by Goldberg and collaborators [3] .

Unfortunately these pioneering works do not give an explicit prescription to transform gravitational waves under rotations and boosts, and to handle their multipolar decomposition. Our main purpose is to fill this gap and to provide examples of the formalism at work, by considering specific problems in numerical relativity and black-hole perturbation theory. We only consider the emission as seen by an observer far away from the source, and we assume

\footnotetext{
* Electronic address: Leonardo.Gualtieri@roma1.infn.it

$\dagger$ Electronic address: Emanuele.Berti@jpl.nasa.gov

$\ddagger$ Electronic address: Vitor.Cardoso@ist.utl.pt

$\S$ Electronic address: Ulrich.Sperhake@uni-jena.de
} 
the spacetime to be asymptotically flat. As discussed in the next section, the spacetime can then be described by a set of coordinates $(u, r, \theta, \phi)$, where $\theta$ and $\phi$ are coordinates on the sphere, $u$ is a retarded time parameter and the limit $r \rightarrow \infty$ (with $u$ finite) represents future null infinity. In this limit $r$ is much larger than $u$ and any translation $\overrightarrow{\delta x}$ of the source: $|\overrightarrow{\delta x}| / r \ll 1,|u| / r \ll 1$. Furthermore, when we consider boosts we will always neglect quantities of order $(V / c)^{2}$, where $V$ is the boost velocity of the source. This approximation is well justified: according to recent results from numerical relativity, black hole mergers produce recoil velocities $V \lesssim 4000 \mathrm{~km} / \mathrm{s}$, i.e. $V / c \lesssim 0.013$ $[23,24,25,26,27]$.

In the remainder of this introduction we list some of the main motivations for our work, and provide a brief summary of our most useful results.

\section{A. Motivations}

The multipolar structure of gravitational radiation is an important element in the problem of detection and parameter estimation from compact binary inspirals. The energy distribution determines which $(l, m)$ components of the radiation are most easily detectable. For a binary inspiral, it has long been believed that the $l=m=2$ mode should dominate the energy emission [28]. Numerical simulations confirm that the $l=m=2$ mode carries $\sim 98 \%$ of the radiation for equal-mass, non-spinning binaries [15]. However, higher modes can carry more than $\sim 10 \%$ of the energy for unequal-mass binaries [16] and/or when the black holes are spinning [18, 19]. Now, recall that the gravitational-wave energy scales with the square of the waves' amplitude. Since SWSHs are normalized over the sphere, this means that the amplitude ratio of the $l=m=3$ and $l=m=2$ modes is typically $\approx 1 / 3$ for generic binaries when different multipolar components are averaged over the sky. Depending on the relative direction of the source and the detector, the enhancement of multipoles with $l>2$ can be even larger (see [29] for a discussion in the context of ringdown detection). For generic binary inspirals, a good phase accuracy on the $l=m=2$ mode is necessary but not sufficient to produce accurate templates: higher multipolar contributions must be taken into account to carefully track the phasing of the waves over many inspiral cycles. One may expect that fewer multipolar components should be sufficient in the early inspiral phase, but this expectation is not confirmed by calculations. For example, by comparing multipolar expansions of the (numerically computed) adiabatic energy flux to post-Newtonian expansions of the flux in the context of extreme mass-ratio inspirals, Ref. [12] showed that the number of multipoles required to achieve a given post-Newtonian accuracy actually increases in the Newtonian regime (i.e. for low orbital frequencies).

The transformation of multipolar decompositions of gravitational radiation under rotations deserves a detailed study for many reasons, some of which we list below.

1) Provide translation rules between numerical codes using different conventions. A pressing motivation for our work is of practical nature. As more accurate and complex numerical waveforms are produced, it is vital to establish a set of conventions between different numerical groups, and rules to translate between different conventions. Binary simulations often differ in subtle details of the numerical settings: for example, some groups initially place the black holes on the $y$-axis, while other groups place them on the $x$-axis. This is obviously a matter of convention, but conventions are important when setting up data formats to be used in LIGO and Virgo data analysis (see e.g. 30] for an attempt in this direction). Similar issues should also be taken into account in collaborative efforts to inject numerical waveforms in the LIGO data stream, such as the Ninja project [31]. One purpose of this paper is to provide a "dictionary" to translate between different conventions by relating multipolar expansions in different frames.

2) Identify the optimal frame to study the merger/ringdown phase in generic spinning binary simulations. Given a generic configuration for a spinning, inspiralling binary, at present there is no reliable analytical method to predict (given the initial data) the orientation of the remnant's spin. We will refer to the frame where the initial orbital angular momentum points in the $z$-direction as the simulation frame, and to the frame where the remnant black hole's spin points in the $z$-direction as the remnant frame.

In the simple (and most-studied) case of non-spinning binaries, a distinction between the simulation frame and the remnant frame is not necessary. The black holes usually orbit on the $x-y$ plane, the initial orbital angular momentum is in the $z$-direction, and angular momentum is radiated only in the $z$-direction. Then the spin of the final black hole will also point in the $z$-direction, and its dimensionless magnitude $j=J / M^{2}$ can be computed by evaluating the $z$-component of the radiated angular momentum and using balance arguments. Since the remnant's spin points in the $z$-direction, a fit of the ringdown frequencies for the dominant SWSH component of the waves in the simulation frame can then be used to verify the accuracy of wave extraction methods [15, 16].

For generic inspirals, however, the system will radiate angular momentum in the $x$ - and $y$-directions as well. The spin of the final black hole will not be aligned with the $z$-axis of the simulation frame. If we (incorrectly) perform the SWSH decomposition in the simulation frame, rather than in the remnant frame, the expansion will mix quasinormal modes with the given value of $l$ and different values of $m$. For this reason, using the wrong frame produces errors in 
the quasinormal frequencies of the final black hole and in ringdown-based estimates of the value of the final angular momentum. Note that modes with different values of $l$ are decoupled, since SWSHs with fixed $l$ and $s$ form an irreducible representation of the rotation group. In Section IV we will illustrate this problem by specific examples, and we will show that rotations can be used to simplify the waveforms: as viewed from the normal to the final black hole's rotation plane, the merger-ringdown radiation in the remnant frame is circularly polarized.

3) Measure the spin direction of the remnant through merger/ringdown waves. The measurement of a single complex quasinormal frequency with Earth-based [29] and space-based interferometers [32] allows a determination of the mass and spin magnitude of the hole resulting from a merger (the $l=m=2$ fundamental quasinormal mode is again expected to be dominant). For Kerr black holes all quasinormal frequencies are uniquely determined by the mass and spin of the hole, and the measurement of the next subdominant mode (typically the fundamental mode with $l=m=3$ ) will provide a test of the Kerr nature of the merger remnant. Since different multipolar components have different angular dependence, a multi-mode detection could even be used to measure the spin direction of the remnant. A detailed analysis of this problem requires the knowledge of the transformation properties of the multipoles under rotations and boosts, and it is an interesting topic for future work.

As argued earlier, boost velocities in binary mergers are typically small $(V / c \lesssim 0.013)$. However the study of boosts is of more than academic interest. In numerical simulations, gravitational radiation should ideally be extracted on a sphere at null infinity. In practice the extraction sphere is usually located at sufficiently large, but finite, distance from the source. In this paper we point out that the distortion of the radiation pattern induced by a large kick of the remnant can induce a mode mixing in the signal. To leading order the amplitude of this mixing is proportional to the kick velocity. The mixing effect will typically be small $\left(V / c \sim 10^{-2}\right.$ for the largest predicted kick velocities), but it may still affect the phasing error of the inspiral waves over long inspiral times. Conversely, the magnitude of the boost-induced mode mixing may set a threshold on the number of high-order post-Newtonian terms we can (or should) compute to get an accurate phasing. Finally, one should not exclude a priori the possibility that the large recession velocities due to Hubble flow may produce observable effects on gravitational waveforms.

Boosts are particularly relevant for the extraction of gravitational waves from the collision of highly relativistic black holes with relatively small impact parameter. This process is of interest as an extreme testing ground of strong-field gravity and in the context of mini black hole production at the Large Hadron Collider, and it is currently under investigation [33].

\section{B. Main results}

For the sake of clarity, and in view of possible generalizations of our results, in the body of this paper we give a detailed account of our calculations. To help the reader, here we provide a short list of results that we consider most useful in phenomenological applications:

- Eq. (3.23) relates the Lorentz transformation of spin-2 SWSHs to the transformation of the Weyl scalar $\Psi_{4}$.

- Eq. (3.28) can be used to obtain spin-2 SWSHs in stereographic coordinates for general values of $l$, and Eq. (3.30) gives closed-form expressions valid for $l=2$ and $l=3$.

- For pure rotations different $l^{\prime} s$ do not couple because SWSHs with given $(s, l)$ form an irreducible representation of the rotation group, and we can consider the transformation laws separately for each value of $l$. The transformation of the harmonics under general rotations, in terms of a constant rotation matrix, is given in Eq. (3.42).

- Eq. (3.53) is one of our main results: it provides an expression for the general rotation matrix, valid for all values of $l$. Eqs. (3.46) and (3.54) list components of the rotation matrix for $l=2$ and $l=3$, respectively. Eqs. (3.49) and (3.56) further specialize these results to frames related by a $\pi / 2$ rotation (i.e. $\theta \rightarrow \theta^{\prime}=\theta-\pi / 2$ ) The transformation under rotations of the Weyl scalar $\Psi_{4}$ and of its complex conjugate has a particularly simple form, which is given in Eqs. (3.59b), 3.60b).

- Eq. (3.58) provides the $l=2$ transformation matrix under non-relativistic boosts, and Eq. (3.65) shows in a special case how boosts produce mode-mixing. As far as we know, this fact has never been pointed out in the existing literature.

- In Section IVA we show that our transformation matrices are consistent with two "classic" calculations. In the first calculation we consider the equal-mass head-on collision of black holes as seen in two frames related by a $\pi / 2$ rotation (i.e. $\theta \rightarrow \theta^{\prime}=\theta-\pi / 2$ ). Then we look at particles falling radially into Schwarzschild black holes along the $z$-axis or along radial, equatorial geodesics. We show that the SWSH components of the stress-energy 
tensor in these two frames are related by proportionality constants which are consistent with our transformation laws.

- In Section IVB we demonstrate that our work can be useful to study the generic merger of unequal-mass, spinning black holes. We suggest a simple recipe to switch from the simulation frame to the remnant frame. Then we show that the merger waveform does indeed look simpler in the remnant frame, because in this frame: (1) ringdown radiation is circularly polarized, and (2) ringdown frequencies are consistent with the final black hole spin predicted from balance arguments. These properties do not apply to the simulation frame.

The paper is organized as follows. In Section $\amalg$ we introduce some basic notions on the BMS group. Section $\amalg$ is the core of this paper, where we derive our main results on the transformation properties of SWSHs and of gravitational radiation under rotations and boosts. Section [IV applies the formalism to specific problems in numerical relativity and perturbation theory, and Section $\mathrm{V}$ discusses some directions for future research. The Appendices contain various technical topics: the expression of non-relativistic boosts in the context of BMS transformations (Appendix A); the description of rotations and boosts in the stereographic representation (Appendix [B]); a discussion of our numerical method to compute the radiated angular momentum for generic, spinning black-hole binaries (Appendix C); and a brief summary of perturbation theory calculations of particles falling into black holes (Appendix D). Unless mentioned otherwise, throughout the paper we use geometrical units $(G=c=1)$.

\section{THE BONDI-METZNER-SACHS GROUP}

To begin with, in this section we briefly recall the main steps of the BMS-group construction. We refer to [2, 21, 34, 35, 36] for a more detailed and rigorous account. Any asymptotically flat spacetime can be described by standard coordinates $(u, r, \theta, \phi)$ defined as follows [21]:

- $u$ is a retarded time parameter, defined by requiring that the hypersurfaces $u=$ constant are null: $g^{\mu \nu} u_{, \mu} u_{, \nu}=0$. These hypersurfaces are the wavefronts of gravitational waves emitted by the source.

- $r$ is an affine parameter of the null geodesics tangent to $l^{\mu}=g^{\mu \nu} u_{, \nu}\left(l^{\mu}\right.$ is also a vector of the Newman-Penrose tetrad).

- $\theta$ and $\phi$ are polar coordinates on the manifolds (diffeomorphic to the 2 -sphere) $r=$ constant, $u=$ constant. Geodesics with constant $(u, \theta, \phi)$ are called "rays," and they are a congruence of geodesics, generators of the hypersurfaces $u=$ constant. The limit $r \rightarrow \infty$ with finite $u$ corresponds to future null infinity, where the gravitational radiation emitted by the source is detected.

- Far away from the source one can define a time coordinate $t=u+r$.

The spacetime is asymptotically flat if the metric tends to the flat space metric sufficiently fast as $r \rightarrow \infty$ (see [21] for further details). This construction allows one to define asymptotic symmetries and then, quoting Sachs, to "separate the kinematics of space-time from the dynamics of the gravitational field at least at spatial infinity" [21]. It is worth noting that such a separation can only be done in asymptotically flat spacetimes, by looking at asymptotic symmetries; otherwise, "in general relativity such a separation is usually impossible" [21].

Asymptotic symmetries are the transformations preserving the asymptotically flat form of the metric, i.e. preserving the above properties. These symmetries form a group: the BMS group. The vector fields generating such transformations satisfy an equation which is weaker than the Killing equation, and coincides with the Killing equation as $r \rightarrow \infty$. The BMS group is defined by the following transformations of the $(u, \theta, \phi)$ coordinates:

$$
\begin{aligned}
\lim _{r \rightarrow \infty} \theta^{\prime} & =\theta^{\prime}(\theta, \phi), \\
\lim _{r \rightarrow \infty} \phi^{\prime} & =\phi^{\prime}(\theta, \phi) \\
\lim _{r \rightarrow \infty} u^{\prime} & =K(\theta, \phi)[u-\alpha(\theta, \phi)],
\end{aligned}
$$

where $\theta^{\prime}(\theta, \phi), \phi^{\prime}(\theta, \phi)$ and $K(\theta, \phi)$ are related by a conformal transformation, i.e.

$$
d \theta^{\prime 2}+\sin ^{2} \theta^{\prime} d \phi^{\prime 2}=K^{2}\left(d \theta^{2}+\sin ^{2} \theta d \phi^{2}\right) .
$$


We follow the convention of Newman and Penrose [2], but note that Ref. [3] uses a different convention: their $K$ is our $K^{-1}$. We can consider Eqs. (2.1) as transformations defined on a three-dimensional manifold described by $(u, \theta, \phi)$, i.e. on the future null hypersurface:

$$
\begin{aligned}
\theta^{\prime} & =\theta^{\prime}(\theta, \phi), \\
\phi^{\prime} & =\phi^{\prime}(\theta, \phi), \\
u^{\prime} & =K(\theta, \phi)[u-\alpha(\theta, \phi)] .
\end{aligned}
$$

The transformations apply to asymptotic quantities defined at future null infinity, which are obtained by expanding tensors (or Newman-Penrose scalars) in powers of $1 / r$ [37]. This approach is particularly meaningful in the study of gravitational-wave emission, since the detector's location is usually a good approximation to future null infinity.

The BMS group is the semidirect sum of the Lorentz group with the group of supertranslations, which are a generalization of translations. The supertranslations are the transformations (2.3) with $\theta^{\prime}=\theta, \phi^{\prime}=\phi$ and $K(\theta, \phi)=1$. A useful illustration of their meaning is given in Ref. [34]. Translations are special cases of supertranslations, with $\alpha(\theta, \phi)=\epsilon_{0}+\epsilon_{i} n_{i}$. Here $i=1,2,3$ and $n_{i}=x_{i} / r=(\sin \theta \cos \phi, \sin \theta \sin \phi, \cos \theta)$ are director cosines.

Lorentz transformations are transformations of the form (2.3) with $\alpha(\theta, \phi)=0$ :

$$
\begin{aligned}
\theta^{\prime} & =\theta^{\prime}(\theta, \phi), \\
\phi^{\prime} & =\phi^{\prime}(\theta, \phi), \\
u^{\prime} & =K(\theta, \phi) u .
\end{aligned}
$$

Strictly speaking, there is no unique way to identify the Lorentz group as a subgroup of the BMS group [2, 38]. Indeed, if $\{L\}$ are the transformations (2.4) and $T(\alpha)$ is a supertranslation with fixed $\alpha$, then the transformations $\left\{T(\alpha) L T^{-1}(\alpha)\right\}$ form another group isomorphic to the Lorentz group. As far as we know, there is no rigorous proof that our choice (2.4) of the Lorentz subgroup is more "natural" than others, but there are arguments supporting this choice. In particular, Ref. [2] suggests that with further, reasonable physical assumptions, the Lorentz group embedded in the BMS group is restricted to $\left\{T(\alpha) L T^{-1}(\alpha)\right\}$ with $\alpha=\epsilon_{0}+\epsilon_{i} n_{i}$ (ordinary translations); the residual freedom, then, corresponds to an arbitrariness in the choice of the origin. Therefore it is not restrictive to assume $\alpha=0$, i.e. to assume that the Lorentz transformations are given by Eq. (2.4). Ordinary rotations are special cases of Lorentz transformations with $K(\theta, \phi)=1$. As discussed in Appendix A boost with (non-relativistic) three-velocity $\vec{V}=\left(V_{1}, V_{2}, V_{3}\right)$ has $K(\theta, \phi)=1+V_{i} n_{i}$.

To study Lorentz transformations, and then conformal transformations on the 2-sphere, it is useful to introduce the stereographic representation, in which the coordinates $(\theta, \phi)$ on the 2 -sphere are mapped on the complex plane by introducing a complex variable

$$
\zeta \equiv \cot \frac{\theta}{2} e^{\mathrm{i} \phi}
$$

Lorentz transformations correspond to mappings $\zeta \rightarrow \zeta^{\prime}$ of the form

$$
\zeta^{\prime}=\frac{a \zeta+c}{b \zeta+d}
$$

where $a, b, c, d$ are complex numbers and we can assume without loss of generality that $a d-b c=1$. The interested reader can find a more detailed discussion in Appendix B]

\section{BMS TRANSFORMATIONS OF GRAVITATIONAL RADIATION}

As we discussed above, $l^{\mu} \equiv g^{\mu \nu} u_{, \nu}$ is a null vector, and $r$ is an affine parameter of the null geodesic tangent to $l^{\mu}$. Following [2, 37, 39], we normalize $r$ by requiring that $l^{\mu}=d x^{\mu} / d r$; furthermore, we take $l^{\mu}$ to be a vector of the Newman-Penrose tetrad. It is important to stress that this is a particular choice of the tetrad [40]: we are fixing the tetrad boost freedom $l^{\mu} \rightarrow \lambda l^{\mu}, n^{\mu} \rightarrow \lambda^{-1} n^{\mu}$ (and then the normalization of $\Psi_{4}$, since for a tetrad boost $\left.\Psi_{4} \rightarrow \lambda^{-2} \Psi_{4}\right)$. Ref. [37] calls this choice the "special coordinate system and associated tetrad", and Ref. [40] calls it the "Bondi tetrad." Most of the literature on the asymptotic behavior of Newman-Penrose quantities and on BMS transformations assumes this tetrad choice. The same normalization has been used by Teukolsky to study perturbations of Kerr black holes [41]. As discussed in [6, 40], recent works dealing with numerical evolutions of the Einstein equations use a tetrad choice such that $l_{\alpha}=u_{, \alpha} / \sqrt{2}[15,16,42$, , 43]. We do not follow the latter convention because Eq. (3.3) below, as derived in [37], requires $l_{\alpha}=u_{, \alpha}$. 


\section{A. Weyl scalar and shear}

Gravitational radiation is described by the Weyl scalar $\Psi_{4}$. The asymptotic limit of $\Psi_{4}$ at future null infinity is closely related to the asymptotic limit of the shear $\sigma$, which is defined in terms of the Newman-Penrose tetrad as

$$
\sigma(u, r, \theta, \phi)=l_{\mu ; \nu} m^{\mu} m^{\nu} .
$$

Indeed, assuming the special coordinate system and the associated tetrad defined above, the asymptotic expansion of $\Psi_{4}$ and $\sigma$ is [37]

$$
\begin{aligned}
\left.\Psi_{4}(r, u, \theta, \phi)\right) & =\frac{\Psi_{4}^{0}(u, \theta, \phi)}{r}+\mathcal{O}\left(r^{-2}\right), \\
\sigma(r, u, \theta, \phi) & =\frac{\sigma^{0}(u, \theta, \phi)}{r^{2}}+\mathcal{O}\left(r^{-4}\right),
\end{aligned}
$$

where

$$
\Psi_{4}^{0}=-\frac{\partial^{2} \bar{\sigma}^{0}}{\partial u^{2}}
$$

and an overbar denotes complex conjugation. To simplify calculations, in the remainder of this paper we will usually consider SWSHs of spin weight $s=2$ and expand the complex-conjugate Weyl scalar as:

$$
\bar{\Psi}_{4}^{0}=e^{-\mathrm{i} \omega u} \sum_{l m}{ }_{2} \psi_{l m 2} Y_{l m}(\theta, \phi),
$$

where we assume a gravitational-wave solution with given frequency $\omega$. In numerical relativity simulations the Weyl scalar is usually expanded in SWSHs of spin-weight $s=-2$. The relevant expansion can easily be obtained by recalling that SWSHs satisfy the property

$$
{ }_{s} Y_{l m}(\theta, \phi)=(-1)^{m+s}{ }_{-s} \bar{Y}_{l-m}(\theta, \phi),
$$

which implies that

$$
\Psi_{4}^{0}=e^{\mathrm{i} \omega u} \sum_{l m}{ }_{-2} \psi_{l m-2} Y_{l m}(\theta, \phi)
$$

with

$$
{ }_{-2} \psi_{l m} \equiv(-1)^{m}{ }_{2} \bar{\psi}_{l-m} .
$$

The first derivative of the shear with respect to retarded time, $\partial \sigma^{0} / \partial u$, is called the news function. Its squared modulus represents the energy density of the gravitational wave: the total energy emitted by the source (corresponding to its mass loss) is [22]

$$
E_{\mathrm{tot}}=\frac{1}{4 \pi} \int\left|\frac{\partial \sigma^{0}}{\partial u}\right|^{2} d u d \Omega
$$

To understand the relation of $\sigma^{0}$ with the gravitational-wave amplitude, let us consider the asymptotic metric in the transverse-traceless (TT) gauge; following the notation of Teukolsky [41],

$$
\Psi_{4}=\frac{1}{2}\left(\ddot{h}_{+}-\mathrm{i} \ddot{h}_{\times}\right)=\frac{\ddot{h}}{2},
$$

where we have defined the complex wave amplitude $h$ in terms of the two polarization modes $h_{+}$and $h_{\times}$as $h \equiv h_{+}-\mathrm{i} h_{\times}$. Assuming again a gravitational-wave solution with given frequency,

$$
h=\frac{1}{r} e^{\mathrm{i} \omega u} \hat{h},
$$

we have

$$
\Psi_{4}=\frac{1}{2} \frac{\partial^{2} h}{\partial u^{2}}
$$


From Eq. (3.3) it follows that

$$
r h=e^{i \omega u} \hat{h}=-2 \bar{\sigma}^{0}
$$

therefore, $\sigma^{0}$ corresponds (modulo proportionality constants) to the wave amplitude $h$.

From Eq. (3.8) we have

$$
\frac{d^{2} E}{d t d \Omega}=\frac{1}{4 \pi}\left|\frac{d^{2} \sigma_{0}}{d u^{2}}\right|=\frac{\omega^{2} r^{2}}{16 \pi}|h|^{2}=\frac{\omega^{2} r^{2}}{16 \pi}\left(\left|h_{+}\right|^{2}+\left|h_{\times}\right|^{2}\right)
$$

which is the well-known expression for the energy carried by a gravitational wave [44]. Notice that

$$
\frac{d^{2} E}{d t d \Omega}=\frac{r^{2}\left|\Psi_{4}^{2}\right|}{4 \pi \omega^{2}}
$$

which is consistent with Ref. [41]. In [15, 16, 42, 43], where $l_{\alpha}=u_{, \alpha} / \sqrt{2}$, the Weyl scalar is $\Psi_{4}=\left(\ddot{h}_{+}-\mathrm{i} \ddot{h}_{\times}\right)$. With their normalization, $d^{2} E / d t d \Omega=r^{2}\left|\Psi_{4}^{2}\right| / 16 \pi \omega^{2}$. The interpretation of the asymptotic shear as the amplitude of the wave in the TT-gauge is also discussed in Ref. [45].

Under a BMS transformation the asymptotic shear transforms as [2, 22]

$$
\sigma^{0 \prime}(u, \theta, \phi)=K^{-1} e^{2 \mathrm{i} \chi}\left(\sigma^{0}(u, \theta, \phi)-\frac{1}{2} \not \not^{2} \alpha\right),
$$

where the operator $\not \partial$, acting on a quantity $\eta$ of spin weight $s$, is defined by

$$
\not \partial \eta=-(\sin \theta)^{s}\left(\frac{\partial}{\partial \theta}+\frac{\mathrm{i}}{\sin \theta} \frac{\partial}{\partial \phi}\right)(\sin \theta)^{-s} \eta
$$

and the angle $\chi$ represents the rotation of the vectors $m^{\mu}, \bar{m}^{\mu}$ of the Newman-Penrose tetrad. For Lorentz transformations $\alpha=0$, and Eq. (3.15) reduces to

$$
\sigma^{0 \prime}(u, \theta, \phi)=K^{-1} e^{2 \mathrm{i} \chi(\theta, \phi)} \sigma^{0}(u, \theta, \phi) .
$$

Incidentally, this relation also holds for ordinary translations, since $\not^{2} \alpha=0$ in this case. The total radiated energy (3.8) transforms as

$$
E_{\mathrm{tot}}^{\prime}=\frac{1}{4 \pi} \int\left|\frac{\partial \sigma^{0 \prime}}{\partial u^{\prime}}\right| d u^{\prime} d \Omega^{\prime}=\frac{1}{4 \pi} \int K^{-4}\left|\frac{\partial \sigma^{0}}{\partial u}\right| K d u K^{2} d \Omega=\frac{1}{4 \pi} \int K^{-1}\left|\frac{\partial \sigma^{0}}{\partial u}\right| d u d \Omega,
$$

with a shift factor $K^{-1}$, as expected. This relation is quite interesting: in the case of a boost, it describes the beaming of the emitted gravitational radiation.

The transformation (3.17) is not yet complete, since $\sigma^{0 \prime}$ is still expressed in the unprimed coordinates $(u, \theta, \phi)$. The complete transformation involves the relation between primed and unprimed coordinates:

$$
\sigma^{0 \prime}\left(u^{\prime}, \theta^{\prime}, \phi^{\prime}\right)=K^{-1} e^{2 \mathrm{i} \chi\left(\theta\left(\theta^{\prime}\right), \phi\left(\phi^{\prime}\right)\right)} \sigma^{0}\left(u\left(u^{\prime}\right), \theta\left(\theta^{\prime}\right), \phi\left(\phi^{\prime}\right)\right) .
$$

For a gravitational wave with frequency $\omega$ we have $\sigma^{0}(u, \theta, \phi)=e^{-i \omega u} \hat{\sigma}^{0}(\theta, \phi)$, and since $u^{\prime}=K u$,

$$
\sigma^{0 \prime}\left(u^{\prime}, \theta^{\prime}, \phi^{\prime}\right)=K^{-1} e^{2 \mathrm{i} \chi\left(\theta\left(\theta^{\prime}\right), \phi\left(\phi^{\prime}\right)\right)} e^{-\mathrm{i} \frac{\omega}{K} u^{\prime}} \hat{\sigma}^{0}\left(\theta\left(\theta^{\prime}\right), \phi\left(\phi^{\prime}\right)\right) .
$$

We see that the frequency of the gravitational wave is blueshifted or redshifted: $\omega \rightarrow \omega^{\prime}=\omega / K$.

The transformation of the asymptotic Weyl scalar $\bar{\Psi}_{4}^{0}$ (we consider the complex conjugate $\bar{\Psi}_{4}^{0}$, with spin 2 , instead of $\Psi_{4}$, with spin -2 , to simplify calculations), related to $\sigma^{0}$ by Eq. (3.3), immediately follows:

$$
\bar{\Psi}_{4}^{0 \prime}\left(u^{\prime}, \theta^{\prime}, \phi^{\prime}\right)=K^{-3} e^{2 \mathrm{i} \chi\left(\theta\left(\theta^{\prime}\right), \phi\left(\phi^{\prime}\right)\right)} \Psi_{4}^{0}\left(u\left(u^{\prime}\right), \theta\left(\theta^{\prime}\right), \phi\left(\phi^{\prime}\right)\right) .
$$

We now expand the complex-conjugate Weyl scalar in SWSHs of spin weight $s=2$, as in Eq. (3.4). Given the transformation of the harmonics

$$
{ }_{2} Y_{l^{\prime} m^{\prime}}\left(\theta^{\prime}(\theta, \phi), \phi^{\prime}(\theta, \phi)\right)=\sum_{l m} D_{l^{\prime} m^{\prime} ; l m} Y_{l m}(\theta, \phi),
$$


the Lorentz transformed Weyl scalar is

$$
\bar{\Psi}_{4}^{0 \prime}\left(u^{\prime}, \theta^{\prime}, \phi^{\prime}\right)=K^{-3} e^{2 \mathrm{i} \chi\left(\theta\left(\theta^{\prime}\right), \phi\left(\phi^{\prime}\right)\right)} e^{-\mathrm{i} \frac{\omega}{K} u^{\prime}} \sum_{l^{\prime} m^{\prime}}{ }_{2} \psi_{l^{\prime} m^{\prime}}^{\prime} Y_{l^{\prime} m^{\prime}}\left(\theta^{\prime}, \phi^{\prime}\right),
$$

with

$$
{ }_{2} \psi_{l^{\prime} m^{\prime}}^{\prime}=\sum_{l m}{ }_{2} \psi_{l m} D_{l m ; l^{\prime} m^{\prime}}^{-1}
$$

In the case of rotations $K=1$ and $D_{l m ; l^{\prime} m^{\prime}}$ is block diagonal in $l$, because spherical harmonics with fixed $l$ and $s$ form irreducible representations of the rotation group. Then the transformation of the Weyl scalar reduces to

$$
\begin{aligned}
\bar{\Psi}_{4}^{0 \prime}\left(u^{\prime}, \theta^{\prime}, \phi^{\prime}\right) & =e^{2 \mathrm{i} \chi\left(\theta\left(\theta^{\prime}\right), \phi\left(\phi^{\prime}\right)\right)} e^{-\mathrm{i} \omega u^{\prime}} \sum_{l m^{\prime}}{ }_{2} \psi_{l m^{\prime} 2}^{\prime} Y_{l m^{\prime}}\left(\theta^{\prime}, \phi^{\prime}\right) \\
{ }_{2} \psi_{l m^{\prime}}^{\prime} & =\sum_{m=-l}^{l}{ }_{2} \psi_{l m} D_{m ; m^{\prime}}^{(l)-1}
\end{aligned}
$$

where we have defined

$$
D_{m m^{\prime}}^{(l)} \equiv D_{l m ; l m^{\prime}}
$$

Therefore, the transformation of the multipolar decomposition of the Weyl scalar under rotations is given by the (inverse) transformation of $s=2 \mathrm{SWSHs}$, modulo an overall phase factor.

We now turn to a detailed treatment of the transformation of SWSHs under BMS transformations. We start with the general case, then specialize to rotations and non-relativistic boosts. Finally we show that there is a simple relation between the transformations of the Weyl scalar components in a SWSH expansion, and the transformations of SWSHs themselves.

\section{B. Transformations of spin-weighted spherical harmonics}

The BMS transformations of SWSHs are discussed in Ref. [3]. Given $s$ and $l \geq|s|$, choose arbitrarily an integer $L \geq l$. Then SWSHs can be written as

$$
{ }_{s} Y_{l m}=\sum_{n_{1}=0}^{L-s} \sum_{n_{2}=0}^{L+s} s B_{l m}^{L n_{1} n_{2}}{ }_{s} Z_{n_{1} n_{2}}^{L}
$$

where ${ }_{s} B_{\underline{l m}}^{L n_{1} n_{2}}$ are constants whose explicit values can be found in Ref. [3] , and ${ }_{s} Z_{n_{1} n_{2}}^{L}$ are properly defined functions of $\zeta$ and $\bar{\zeta}$. This equation can be rewritten in a simpler form:

$$
{ }_{2} Y_{l m}=(-1)^{m} \sqrt{\frac{2 l+1}{4 \pi}} \sqrt{\frac{(l+m) !(l-m) !}{(l+2) !(l-2) !}} \sum_{n}(-1)^{n}\left(\begin{array}{c}
l-2 \\
n
\end{array}\right)\left(\begin{array}{c}
l+2 \\
l-m-n
\end{array}\right) Z_{l m}^{n}
$$

where $\max (0,-2-m) \leq n \leq l-\max (m, 2)$ and we have defined

$$
Z_{l m}^{n} \equiv(\zeta \bar{\zeta})^{l-2-n} \bar{\zeta}^{2-m}(1+\zeta \bar{\zeta})^{-l}
$$

This expression is consistent with Eq. (4.13) of Ref. [3] if we correct for an erroneous factor of $(-1)^{m}$ in their Eq. $(2.12)$. For $l=2$ and $l=3$ the SWSHs reduce to

$$
\begin{aligned}
& { }_{2} Y_{2 m}=(-1)^{m} \sqrt{\frac{30}{\pi}} \frac{1}{\sqrt{(2+m) !(2-m) !}} Z_{m}, \\
& { }_{2} Y_{3 m}=(-1)^{m+1} \sqrt{\frac{210}{\pi}}\left[\frac{1-\delta_{m, 3}}{(2-m) !} \sqrt{\frac{(3-m) !}{(3+m) !}} Z_{m}^{(+)}-\frac{1-\delta_{m,-3}}{(2+m) !} \sqrt{\frac{(3+m) !}{(3-m) !}} Z_{m}^{(-)}\right]
\end{aligned}
$$


where we have defined

$$
\begin{array}{rlrl}
Z_{m} & \equiv{ }_{2} Z_{0 m+2}^{2}=\frac{1}{(1+\zeta \bar{\zeta})^{2}} \bar{\zeta}^{2-m}=\left(\sin \frac{\theta}{2}\right)^{2+m}\left(\cos \frac{\theta}{2}\right)^{2-m} e^{\mathrm{i} m \phi} e^{-2 \mathrm{i} \phi}, \quad(-2 \leq m \leq 2), \\
Z_{m}^{(+)} \equiv{ }_{2} Z_{1 m+3}^{3}=\frac{1}{(1+\zeta \bar{\zeta})^{3}} \bar{\zeta}^{2-m}=\left(\sin \frac{\theta}{2}\right)^{4+m}\left(\cos \frac{\theta}{2}\right)^{2-m} e^{\mathrm{i} m \phi} e^{-2 \mathrm{i} \phi}, \quad(-3 \leq m \leq 2), \\
Z_{m}^{(-)} \equiv{ }_{2} Z_{0 m+2}^{3}=\frac{\zeta \bar{\zeta}}{(1+\zeta \bar{\zeta})^{3}} \bar{\zeta}^{2-m}=\left(\sin \frac{\theta}{2}\right)^{2+m}\left(\cos \frac{\theta}{2}\right)^{4-m} e^{\mathrm{i} m \phi} e^{-2 \mathrm{i} \phi}, \quad(-2 \leq m \leq 3) .
\end{array}
$$

The factor $e^{-2 \mathrm{i} \phi}$ is due to different conventions in defining SWSHs, corresponding to different choices of the NewmanPenrose tetrad (see footnote 6 of [3]). We have checked that Eqs. (3.28) and (3.30) are consistent with the standard definition of SWSHs in terms of ordinary spherical harmonics:

$$
{ }_{2} Y_{l m}=\frac{1}{\sqrt{(l-1) l(l+1)(l+2)}}\left(\partial_{\theta}^{2}-\cot \theta \partial_{\theta}+\frac{m^{2}}{\sin ^{2} \theta}-\frac{2 m}{\sin \theta}\left(\partial_{\theta}-\cot \theta\right)\right) Y_{l m},
$$

where we have followed the conventions of [3], adopted also in recent numerical simulations [15, 16, 42, 43]. The only difference between Eq. (3.32) and our expressions is a global factor of $e^{-2 i \phi}$, which does not depend on $m$ and is therefore irrelevant. The $s=-2$ harmonics can be obtained by complex conjugation, using Eq. (3.5).

Let us focus for the time being on $l=2$. In stereographic coordinates (see Appendix B) a Lorentz transformation maps $\zeta \rightarrow \zeta^{\prime}$ as in Eq. (2.6), and

$$
Z_{m^{\prime}}^{\prime}=K^{2} e^{2 \mathrm{i} \chi} \frac{1}{(1+\zeta \bar{\zeta})^{2}}(\bar{a} \bar{\zeta}+\bar{c})^{2-m^{\prime}}(\bar{b} \bar{\zeta}+\bar{d})^{2+m^{\prime}}=K^{2} e^{2 \mathrm{i} \chi} \sum_{m=-2}^{2} F_{m^{\prime} m} Z_{m}, \quad(-2 \leq m \leq 2),
$$

where the constants $F_{m^{\prime} m}$ are given by the expansion coefficients of polynomials of the form

$$
(\bar{a} \bar{\zeta}+\bar{c})^{2-m^{\prime}}(\bar{b} \bar{\zeta}+\bar{d})^{2+m^{\prime}}=\sum_{m=-2}^{2} F_{m^{\prime} m} \bar{\zeta}^{2-m}, \quad\left(-2 \leq m^{\prime} \leq 2\right) .
$$

Replacing Eqs. (3.33) and (3.34) in Eq. (3.30a) we find the transformation of spin-2 SWSHs with $l=2$ and its inverse:

$$
\begin{aligned}
{ }_{2} Y_{2 m^{\prime}}\left(\theta^{\prime}, \phi^{\prime}\right) & =K^{2}(\theta, \phi) e^{2 \mathrm{i} \chi(\theta, \phi)} \sum_{m=-2}^{2} A_{m^{\prime} m}{ }_{2} Y_{2 m}(\theta, \phi), \\
{ }_{2} Y_{2 m}(\theta, \phi) & =K^{-2} e^{-2 \mathrm{i} \chi\left(\theta^{\prime}, \phi^{\prime}\right)} \sum_{m^{\prime}=-2}^{2} A_{m m^{\prime}}^{(2)-1}{ }_{2} Y_{2 m^{\prime}}\left(\theta^{\prime}, \phi^{\prime}\right),
\end{aligned}
$$

where

$$
A_{m^{\prime} m}^{(2)} \equiv(-1)^{m+m^{\prime}} \sqrt{\frac{(2+m) !(2-m) !}{\left(2+m^{\prime}\right) !\left(2-m^{\prime}\right) !}} F_{m^{\prime} m}
$$

The constant coefficients of the inverse matrix $A_{m m^{\prime}}^{(2)-1}$ can be derived using the inverse Lorentz transformation

$$
\zeta=\frac{d \zeta^{\prime}-c}{-b \zeta^{\prime}+a}
$$

or the identity

$$
\sum_{m=-2}^{2} A_{m^{\prime} m}^{(2)} A_{m m^{\prime \prime}}^{(2)-1}=\delta_{m^{\prime} m^{\prime \prime}}
$$

For $l>2$ the transformation of the harmonics is more involved. Eq. (3.36) can easily be generalized only in the case of pure rotations, due to the decoupling of harmonics with different values of $l$, but the general Lorentz transformations are more difficult to work out explicitly.

Below we discuss two special cases in which the formalism simplifies considerably: pure rotations and non-relativistic boosts with $|V| \ll 1$. 


\section{Transformations under rotations}

Since spin- $s$ SWSHs with a given value of $l$ are a representation of the rotation group, for pure rotations we get

$$
{ }_{2} Y_{l m^{\prime}}\left(\theta^{\prime}, \phi^{\prime}\right)=\sum_{m=-l}^{l} D_{m^{\prime} m}^{(l)} Y_{l m}(\theta, \phi) .
$$

where $D_{m^{\prime} m}^{(l)}$ has been defined in Eq. (3.26). Since $K=1$ for pure rotations, Eq. (3.35) gives

$$
D_{m^{\prime} m}^{(2)}=e^{2 \mathrm{i} \chi} A_{m^{\prime} m}^{(2)},
$$

with $A_{m^{\prime} m}^{(2)}$ a constant matrix. As we discuss below, this property holds for all $l$ 's: $D_{m^{\prime} m}^{(l)}=e^{2 \mathrm{i} \chi} A_{m^{\prime} m}^{(l)}$, and therefore

$$
{ }_{2} Y_{l m^{\prime}}\left(\theta^{\prime}, \phi^{\prime}\right)=e^{2 \mathrm{i} \chi} \sum_{m} A_{m^{\prime} m}^{(l)} Y_{l m}(\theta, \phi),
$$

with $A_{m^{\prime} m}^{(l)}$ a constant matrix.

By appropriately choosing the primed frame, a general rotation can be reduced to a rotation mapping any vector (such as the total angular momentum) to the $z^{\prime}$-axis:

$$
\left(J_{x}, J_{y}, J_{z}\right)=\left(J \sin \theta_{0} \cos \phi_{0}, J \sin \theta_{0} \sin \phi_{0}, J \cos \theta_{0}\right) \rightarrow(0,0, J) .
$$

As discussed in Appendix B the stereographic representation of such a rotation has the form

$$
a=c_{0} e^{-\mathrm{i} \frac{\phi_{0}}{2}}, \quad b=-s_{0} e^{-\mathrm{i} \frac{\phi_{0}}{2}}, \quad c=s_{0} e^{\mathrm{i} \frac{\phi_{0}}{2}}, \quad d=c_{0} e^{\mathrm{i} \frac{\phi_{0}}{2}},
$$

where we have used the short-hand notation

$$
c_{0} \equiv \cos \left(\theta_{0} / 2\right), \quad s_{0} \equiv \sin \left(\theta_{0} / 2\right) .
$$

The corresponding transformation matrix for $l=2$ SWSHs with $s=2, A_{m^{\prime} m}^{(2)}$, is

$$
e^{\mathrm{i} m \phi_{0}} A_{m^{\prime} m}^{(2)}=\left(\begin{array}{ccccc}
c_{0}^{4} & -2 c_{0}^{3} s_{0} & \sqrt{6} c_{0}^{2} s_{0}^{2} & -2 c_{0} s_{0}^{3} & s_{0}^{4} \\
2 c_{0}^{3} s_{0} & c_{0}^{2}\left(1-4 s_{0}^{2}\right) & \sqrt{6} s_{0} c_{0}\left(1-2 c_{0}^{2}\right) & 4 s_{0}^{2}\left(c_{0}^{2}-1 / 4\right) & -2 c_{0} s_{0}^{3} \\
\sqrt{6} c_{0}^{2} s_{0}^{2} & -\sqrt{6} s_{0} c_{0}\left(1-2 c_{0}^{2}\right) & 1-6 c_{0}^{2} s_{0}^{2} & \sqrt{6} s_{0} c_{0}\left(1-2 c_{0}^{2}\right) & \sqrt{6} c_{0}^{2} s_{0}^{2} \\
2 c_{0} s_{0}^{3} & 4 s_{0}^{2}\left(c_{0}^{2}-1 / 4\right) & -\sqrt{6} s_{0} c_{0}\left(1-2 c_{0}^{2}\right) & c_{0}^{2}\left(1-4 s_{0}^{2}\right) & -2 s_{0} c_{0}^{3} \\
s_{0}^{4} & 2 c_{0} s_{0}^{3} & \sqrt{6} c_{0}^{2} s_{0}^{2} & 2 c_{0}^{3} s_{0} & c_{0}^{4}
\end{array}\right) .
$$

Here and below, whenever we write the transformation in matrix form, different rows correspond to increasing values of $m^{\prime}(=-l, \ldots, l)$, and different columns correspond to increasing values of $m(=-l, \ldots, l)$.

As a useful special case, consider the transformation of the $l=2$ harmonics under a counterclockwise rotation by $\pi / 2$ around the $x_{2}$-axis. In stereographic coordinates this transformation reduces to

$$
\zeta^{\prime}=\frac{\frac{\zeta}{\sqrt{2}}+\frac{1}{\sqrt{2}}}{-\frac{\zeta}{\sqrt{2}}+\frac{1}{\sqrt{2}}},
$$

so Eq. (3.34) becomes

$$
\frac{1}{4}(-1)^{m^{\prime}}(\bar{\zeta}-1)^{2+m^{\prime}}(\bar{\zeta}+1)^{2-m^{\prime}}=F_{m^{\prime} m} \bar{\zeta}^{2-m} .
$$

The corresponding coefficients $A_{m^{\prime} m}^{(2)}$ are

$$
A_{m^{\prime} m}^{(2)}=\frac{1}{4}\left(\begin{array}{ccccc}
1 & -2 & \sqrt{6} & -2 & 1 \\
2 & -2 & 0 & 2 & -2 \\
\sqrt{6} & 0 & -2 & 0 & \sqrt{6} \\
2 & 2 & 0 & -2 & -2 \\
1 & 2 & \sqrt{6} & 2 & 1
\end{array}\right)
$$


The transformation under rotations of spin-2 SWSHs with $l>2$ is sligthly more involved. Under a rotation, the functions $Z_{l m}^{n}$ defined in Eq. (3.29) transform to

$$
Z_{l m^{\prime}}^{\prime n^{\prime}}=\frac{e^{2 \mathrm{i} \chi}}{(1+\zeta \bar{\zeta})^{l}}(\bar{a} \bar{\zeta}+\bar{c})^{l-m^{\prime}-n^{\prime}}(\bar{b} \bar{\zeta}+\bar{d})^{2+m^{\prime}+n^{\prime}}(a \zeta+c)^{l-2-n^{\prime}}(b \zeta+d)^{n^{\prime}}=e^{2 \mathrm{i} \chi} \sum_{m n} F_{m^{\prime} m}^{n^{\prime} n} Z_{l m}^{n},
$$

which defines (implicitly) the constant coefficients $F_{m^{\prime} m}^{n^{\prime} n}$. In Eq. (3.50) the indices $n, n^{\prime}$ are defined in the ranges

$$
\max (0,-2-m) \leq n \leq l-\max (m, 2), \quad \max \left(0,-2-m^{\prime}\right) \leq n^{\prime} \leq l-\max \left(m^{\prime}, 2\right),
$$

respectively.

Correspondingly, the spherical harmonics transform to

$$
\begin{aligned}
{ }_{2} Y_{l m^{\prime}}^{\prime} & =(-1)^{m^{\prime}} \sqrt{\frac{2 l+1}{4 \pi}} \sqrt{\frac{\left(l+m^{\prime}\right) !\left(l-m^{\prime}\right) !}{(l+2) !(l-2) !}} \sum_{n^{\prime}}(-1)^{n^{\prime}}\left(\begin{array}{c}
l-2 \\
n^{\prime}
\end{array}\right)\left(\begin{array}{c}
l+2 \\
l-m^{\prime}-n^{\prime}
\end{array}\right)\left(e^{2 \mathrm{i} \chi} \sum_{m n} F_{m^{\prime} m}^{n^{\prime} n} Z_{l m}^{n}\right) \\
& =e^{2 \mathrm{i} \chi}(-1)^{m} \sqrt{\frac{2 l+1}{4 \pi}} \sqrt{\frac{(l+m) !(l-m) !}{(l+2) !(l-2) !}} \sum_{n}(-1)^{n}\left(\begin{array}{c}
l-2 \\
n
\end{array}\right)\left(\begin{array}{c}
l+2 \\
l-m-n
\end{array}\right) A_{m^{\prime} m}^{(l)} Z_{l m}^{n} .
\end{aligned}
$$

where we defined

$$
A_{m^{\prime} m}^{(l)} \equiv \sum_{n^{\prime}}(-1)^{m+m^{\prime}+n+n^{\prime}} \sqrt{\frac{\left(l+m^{\prime}\right) !\left(l-m^{\prime}\right) !}{(l+m) !(l-m) !}} \frac{\left(\begin{array}{c}
l-2 \\
n^{\prime}
\end{array}\right)\left(\begin{array}{c}
l+2 \\
l-m^{\prime}-n^{\prime}
\end{array}\right)}{\left(\begin{array}{c}
l-2 \\
n
\end{array}\right)\left(\begin{array}{c}
l+2 \\
l-m-n
\end{array}\right)} F_{m^{\prime} m}^{n^{\prime} n} .
$$

Remarkably, the right-hand side of Eq. (3.53) does not seem to depend on the index $n$. This property implies Eq. (3.42), which shows explicitly that SWSHs with fixed $l$ and $s$ form an irreducible representation of the rotation group, as expected from group-theoretical considerations. We do not have an analytic proof that $A_{m^{\prime} m}^{(l)}$ is independent of $n$. However we checked this property for $l \leq 10$ and all values of $m, m^{\prime}$ (which include all cases of interest for gravitational wave detection), and we expect it to hold in general.

The coefficients $A_{m^{\prime} m}^{(l)}$ can explicitly be computed case-by-case from Eq. (3.53). Note that the phase factor $e^{2 i \chi}$ is independent of $l$. As we will show, this phase factor cancels out in the transformation of the Weyl scalars.

For reference, let us evaluate explicitly the components of the rotation matrix $A_{m^{\prime} m}^{(l)}$ for $l=3$. For a general rotation by angles $\left(\theta_{0}, \phi_{0}\right)$ the transformation matrix $A_{m^{\prime} m}^{(3)}$ is

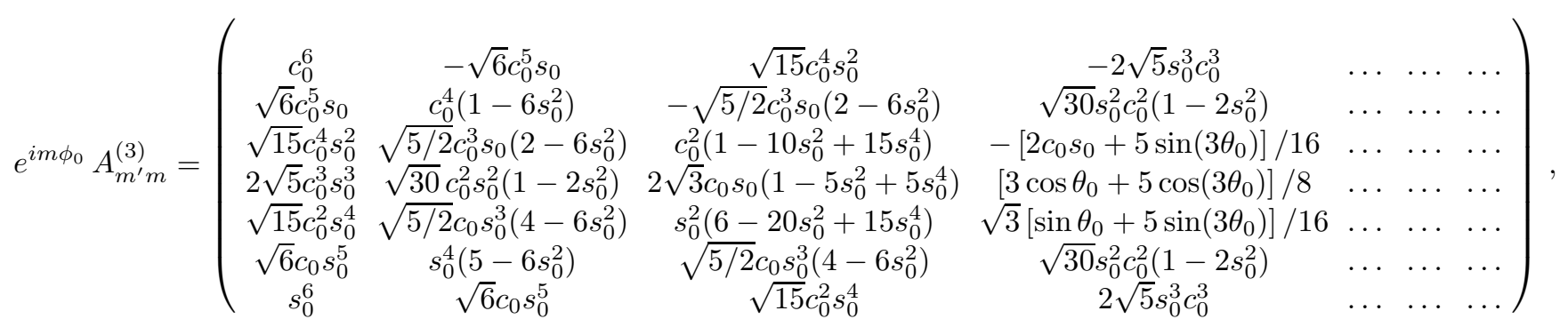

where again we have used the short-hand notation defined in Eq. (3.45). The missing components can be obtained with the help of the general relation

$$
A_{m^{\prime} m}^{(l)}=(-1)^{m+m^{\prime}} \bar{A}_{-m^{\prime}-m}^{(l)} .
$$

In the special case of a $\pi / 2$ counterclockwise rotation around the $x_{2}$-axis, from these relations and Eq. (3.47) we get

$$
A_{m^{\prime} m}^{(3)}=\frac{1}{8}\left(\begin{array}{ccccccc}
1 & -\sqrt{6} & \sqrt{15} & -2 \sqrt{5} & \sqrt{15} & -\sqrt{6} & 1 \\
\sqrt{6} & -4 & \sqrt{10} & 0 & -\sqrt{10} & 4 & -\sqrt{6} \\
\sqrt{15} & -\sqrt{10} & -1 & 2 \sqrt{3} & -1 & -\sqrt{10} & \sqrt{15} \\
2 \sqrt{5} & 0 & -2 \sqrt{3} & 0 & 2 \sqrt{3} & 0 & -2 \sqrt{5} \\
\sqrt{15} & \sqrt{10} & -1 & -2 \sqrt{3} & -1 & \sqrt{10} & \sqrt{15} \\
\sqrt{6} & 4 & \sqrt{10} & 0 & \sqrt{10} & -4 & -\sqrt{6} \\
1 & \sqrt{6} & \sqrt{15} & 2 \sqrt{5} & \sqrt{15} & \sqrt{6} & 1
\end{array}\right) .
$$




\section{Transformations under non-relativistic boosts}

In the case of boosts, Appendix $\mathrm{B}$ shows that the conformal factor is $K=1+V_{i} n_{i}$ to linear order in the boost velocity. At the same order, the transformation in the stereographic representation is described by the parameters

$$
a=1-\frac{V_{3}}{2}, \quad b=-\frac{V_{-}}{2}, \quad c=-\frac{V_{+}}{2}, \quad d=1+\frac{V_{3}}{2},
$$

where we have defined $V_{ \pm} \equiv V_{1} \pm i V_{2}$, and $V_{i} \ll 1$ is the boost velocity along the $x_{i}$-axis (see Appendix B). The components $A_{m^{\prime} m}^{(2)}$ can now be determined from Eqs. (3.34) and (3.37). To first order in $V_{i}$ they read

$$
A_{m^{\prime} m}^{(2)}=\left(\begin{array}{ccccc}
1-2 V_{3} & V_{+} & 0 & 0 & 0 \\
V_{-} & 1-V_{3} & \sqrt{3 / 2} V_{+} & 0 & 0 \\
0 & \sqrt{3 / 2} V_{-} & 1 & \sqrt{3 / 2} V_{+} & 0 \\
0 & 0 & \sqrt{3 / 2} V_{-} & 1+V_{3} & V_{+} \\
0 & 0 & 0 & V_{-} & 1+2 V_{3}
\end{array}\right) .
$$

We stress that, although only $l=2$ harmonics formally appear in the expansion (3.35), for a boost there is no true decoupling in $l$, since the conformal factor $K$ has a non-trivial dependence on $\theta$ and $\phi$. Harmonics with $l>2$ would appear if we expanded in constant coefficients, as in Eq. (3.22).

\section{Transformations of the Weyl scalar}

In order to find the Lorentz transformation of the Weyl scalar $\Psi_{4}$, as given in Eqs. (33.23) and (3.24), we need to determine the inverse transformation of spin-2 SWSHs, i.e. the coefficients $D_{l m ; l^{\prime} m^{\prime}}^{-1}$. We will do so in the following.

\section{Transformations under rotations}

Under rotations, using Eqs. (3.25a) and (3.42), Eq. (3.23) reduces to

$$
\begin{aligned}
\bar{\Psi}_{4}^{0 \prime}\left(u^{\prime}, \theta^{\prime}, \phi^{\prime}\right) & =e^{-\mathrm{i} \omega u} \sum_{l m^{\prime}}{ }_{2} \psi_{l m^{\prime}}^{\prime} Y_{l m^{\prime}}\left(\theta^{\prime}, \phi^{\prime}\right) \\
{ }_{2} \psi_{l m^{\prime}}^{\prime} & =\sum_{m}{ }_{2} \psi_{l m} A_{m m^{\prime}}^{(l)-1} .
\end{aligned}
$$

It is worth noting that the phase factors $e^{ \pm 2 i \chi}$ cancel, and the transformation under rotations of the coefficients of the multipolar expansion of $\bar{\Psi}_{4}$ coincides with the inverse transformation of spin-2 SWSHs.

The transformation laws for the standard decomposition of the Weyl scalar $\Psi_{4}$ in $s=-2$ harmonics can be obtained with the help of relations (3.5), (3.7). However, a simpler relation emerges if one uses Eq. (3.55). Using the complex conjugate of Eqs. (3.59b) and (3.55) one can show that

$$
\begin{aligned}
& \Psi_{4}^{0 \prime}\left(u^{\prime}, \theta^{\prime}, \phi^{\prime}\right)=e^{\mathrm{i} \omega u} \sum_{l m^{\prime}}-2 \psi_{l m^{\prime}-2}^{\prime} Y_{l m^{\prime}}\left(\theta^{\prime}, \phi^{\prime}\right), \\
& { }_{-2} \psi_{l m^{\prime}}^{\prime}=\sum_{m}-2 \psi_{l m} A_{m m^{\prime}}^{(l)-1}
\end{aligned}
$$

This means that the coefficients of $s=-2$ and $s=+2$ transform with the same representation. Some applications of Eq. (3.60b) to numerical relativity simulations of black hole mergers are discussed in Section IV.

\section{Transformations under boosts}

The case of pure boosts is more complex than a pure rotation. The conformal factor $K$ is now a function of $\theta$ and $\phi$, so it is not trivial to extract the transformation law of the coefficients ${ }_{2} \psi_{l m}$ from Eq. (3.23).

Quite interestingly, the factor $K(\theta, \phi)$ couples harmonics with different values of $l$. To see how this coupling comes about and how boosts affect the mode decomposition, let us consider a gravitational wave which in the original frame 
consists of an $(l=2, m=0)$ mode only. If we move to a frame which is boosted by $V_{3}$ along the $x_{3}$-axis and we neglect the phase factor $e^{-i \frac{\omega}{K} u^{\prime}}$ (e.g. by considering wavefronts for which $u^{\prime}=0$ ), from Eq. (3.23) we get

$$
\bar{\Psi}_{4}^{0 \prime}\left(u^{\prime}, \theta^{\prime}, \phi^{\prime}\right) \approx\left(1-5 V_{3} \cos \theta^{\prime}\right){ }_{2} \psi_{20} \sum_{m^{\prime}} A_{0 m^{\prime}}^{(2)-1}{ }_{2} Y_{2 m^{\prime}}\left(\theta^{\prime}, \phi^{\prime}\right)
$$

where we expanded $K^{-5}=\left(1+V_{3} \cos \theta\right)^{-5} \simeq 1-5 V_{3} \cos \theta$. The inverse transformation matrix, ignoring second-order terms, is

$$
A_{m m^{\prime}}^{(2)-1}=\left(\begin{array}{ccccc}
1-2 V_{3} & 0 & 0 & 0 & 0 \\
0 & 1+V_{3} & 0 & 0 & 0 \\
0 & 0 & 1 & 0 & 0 \\
0 & 0 & 0 & 1-V_{3} & 0 \\
0 & 0 & 0 & 0 & 1-2 V_{3}
\end{array}\right)
$$

If we equate Eq. (3.61) to Eq. 3.59a) we get

$$
\sum_{l m}{ }_{2} \psi_{l m}^{\prime}{ }_{2} Y_{l m}\left(\theta^{\prime}, \phi^{\prime}\right)={ }_{2} \psi_{20}\left(1-5 V_{3} \cos \theta^{\prime}\right){ }_{2} Y_{20}\left(\theta^{\prime}, \phi^{\prime}\right)={ }_{2} \psi_{20}\left[{ }_{2} Y_{20}\left(\theta^{\prime}, \phi^{\prime}\right)-\frac{5}{\sqrt{7}} V_{3} Y_{30}\left(\theta^{\prime}, \phi^{\prime}\right)\right]
$$

where we used the property

$$
\cos \theta_{2}^{\prime} Y_{20}\left(\theta^{\prime}, \phi^{\prime}\right)=\frac{1}{\sqrt{7}}{ }_{2} Y_{30}\left(\theta^{\prime}, \phi^{\prime}\right) .
$$

Therefore, the boost produces an $l=3$ component: the nonvanishing components of the multipolar expansion are

$$
{ }_{2} \psi_{20}^{\prime}={ }_{2} \psi_{20}, \quad{ }_{2} \psi_{30}^{\prime}=-\frac{5}{\sqrt{7}} V_{3}{ }_{2} \psi_{20} \text {. }
$$

An $l=2$ waveform is seen as distorted in the boosted frame, with contributions also from $l=3$. If we took into account higher powers of $V$ we would find that higher multipoles would also be excited.

Some considerations on the physical relevance of this coupling are in order. For unequal-mass spinning black hole binaries, the final black hole can be kicked with velocities as large as $4000 \mathrm{~km} / \mathrm{s}$ [23, 24, 25, 27]. In the center-ofmass frame, numerical simulations (and possibly also post-Newtonian expansions) will see an $l=3$ component due entirely to the fact that the black hole is boosted. The $l=3$ component can be as large as $V_{3} \sim 10^{-2}$ times the $l=2$ component. Boost-induced multipole coupling will certainly be relevant in small impact-parameter collisions of ultrarelativistic black holes: in this case the velocity is a sizeable fraction of the speed of light, and the energy transfer between modes can have important consequences. A detailed study of this problem will be reported elsewhere.

\section{APPLICATION TO NUMERICAL SIMULATIONS AND BLACK HOLE PERTURBATION THEORY}

In this section we apply the tools developed in the rest of the paper to numerical simulations of binary black-hole mergers and to a classical problem in black-hole perturbation theory. We examine in detail two scenarios.

In the first scenario, we consider two possible physical situations: the head-on collision of equal-mass, non-spinning black holes in numerical relativity, and the infall of a point particle into a Schwarzschild black hole in perturbation theory. We study the collision in two different frames: (1) a frame where the collision (or infall) occurs along the $z$-axis, making maximal use of the symmetries of the system, and (2) a frame where the collision occurs in the equatorial plane (along the $x$-axis, for the equal-mass case). The two frames are obviously related by a $\pi / 2$ rotation: $\theta \rightarrow \theta^{\prime}=\theta-\pi / 2$. Our main purpose is to show in a relatively simple but non-trivial setting that our formalism is consistent with the predictions of numerical simulations and of black-hole perturbation theory.

In the second scenario we consider a more general case: the merger of unequal-mass, spinning black holes in a socalled "superkick" configuration [23, 24, 25, 27]. This configuration is general enough to provide a good case study for generic, unequal-mass mergers of spinning black holes. Our main goal in this exercise is to stress the importance, in the context of generic mergers, of relating two reference frames: the "simulation frame" (where the binary initially orbits on the $x-y$ plane, so that the orbital angular momentum initially points in the $z$-direction) and the "remnant frame" (defined as the frame where the final black hole's spin is aligned with the $z$-axis). To a good level of approximation, if we ignore the (relatively small) boost, the two frames are related by a rotation. We suggest a simple method to estimate the angles $\left(\theta_{0}, \phi_{0}\right)$ describing this rotation. Then we argue that the analysis of merger waveforms is more "natural" in the remnant frame. More specifically, we show that (i) the merger radiation, as seen from the $z$-axis, is circularly polarized in the remnant frame, but not in the simulation frame, and (ii) ringdown frequencies provide reasonable estimates of the final black-hole spin in the remnant frame, but not in the simulation frame. 


\section{A. Head-on collisions of non-spinning black holes}

Let us start by discussing how rotations affect the description of two "simple," highly symmetric processes leading to the generation of gravitational waves: the head-on collisions of equal-mass, non-spinning black holes in numerical relativity, and the infall of a point particle into a Schwarzschild black hole in perturbation theory.

\section{Equal mass head-on collisions in numerical relativity}

For an equal mass head-on collision of non-spinning holes along the $z$-axis, by symmetry, only modes with $m=0$ are excited (see [43] and references therein). In Ref. [46] we studied the transition from circular inspirals to head-on collisions by considering a sequence of eccentric inspirals. Initially the black holes were placed on the $x$-axis. Along the sequence, we fixed the system's binding energy. The (equal and opposite) linear momenta on the two holes were directed along the $y$-axis, and gradually reduced from the value $P=P_{\text {circ }}$ required to produce a quasi-circular inspiral down to $P=0$. In the limit $P=0$ the black holes collide head-on along the $x$-axis. By analyzing the resulting radiation we observed that it contains components with $(l=2, m= \pm 2)$ and $(l=2, m=0)$, and that these components have roughly comparable amplitude (see top left panel in Fig. 2 of [46]). Now we are in a position to predict quantitatively the relative amplitude of these components. In the frame where the holes collide along the $z$-axis, we should only have a component with $(l=2, m=0)$. By colliding the holes along the $x$-axis, we are effectively performing a $\pi / 2$ rotation in the $\theta$-direction. But then from Eq. (3.49) we immediately see that the $(l=2, m=2)$ and $(l=2, m=0)$ wave amplitudes should be proportional to each other, with a proportionality factor of $-\sqrt{6} / 2$. In Fig. 1 we show that this prediction is in excellent agreement with the numerical simulations. The error (short-dashed, green curve) is orders of magnitude below the wave signal except for those times when the GWs are so weak that $\Psi_{4}$ is dominated by numerical noise; that is, during the early inspiral, once the junk radiation has left the system, and in the late ringdown stages. Ref. [47] independently derived a similar result in the context of head-on collisions of boson stars.

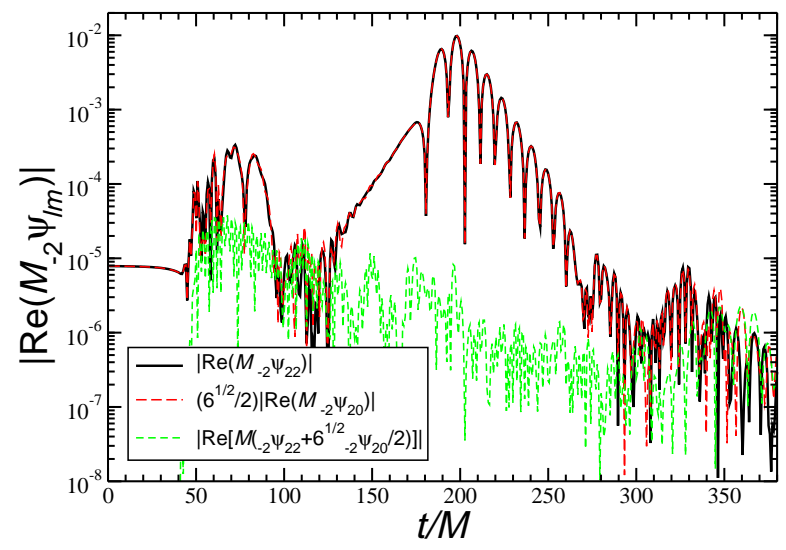

FIG. 1: Check of the transformation law for the $l=2$ modes of the head-on collisions studied in [46]. To better quantify the error, the short-dashed (green) line shows $\left|\operatorname{Re}\left[M\left({ }_{-2} \psi_{22}+\sqrt{6}_{-2} \psi_{20} / 2\right)\right]\right|$. This quantity should be zero, and indeed it is typically much smaller than the corresponding amplitudes, with the exception of the early inspiral and late ringdown phases.

\section{Infall of particles in black-hole perturbation theory}

The previous discussion referred to equal-mass black hole collisions. The limit where one black hole is much smaller than the other $\left(M_{1} \ll M_{2}\right)$ is also of great interest, since it can be described within black hole perturbation theory. In this framework the small black hole is considered as a perturbation in the geometry of the large black hole, and one can expand Einstein's equations to first order in the parameter $M_{1} / M_{2}$. The resulting system of equations can be further reduced to a single ordinary differential equation with a source term $\mathcal{L}$. The functional form of the source 
term depends of course on the frame chosen to do the calculation. In particularly symmetric situations (e.g. for the radial infall of a small body into a more massive black hole) the source term has a simple analytical form. For this reason, the radial infall of a point particle into a black hole is a good testing ground for the formalism we developed in this paper. We consider the source term in two frames: one where the infall occurs along the symmetry axis (so that only modes with $m=0$ are non-vanishing) and a frame rotated by $\pi / 2$, so that the collision occurs in the equatorial plane.

Details of the formalism are discussed in Appendix D. Here we only report the main result: for particles falling along the axis and in the equatorial plane the source terms are given by Eqs. (D10)-(D11) and (D14a)-(D14b), respectively. Since we work in linear perturbation theory the waveforms are proportional to $\mathcal{L}$, and therefore we infer that

$$
\frac{{ }_{-2} \psi_{22}^{\text {equator }}}{{ }_{-2} \psi_{20}^{\text {equator }}}=-\frac{\sqrt{6}}{2}, \quad \frac{{ }_{-2} \psi_{20}^{\text {axis }}}{{ }_{-2} \psi_{20}^{\text {equator }}}=2, \quad \frac{{ }_{2} \psi_{3 \pm 3}^{\text {equator }}}{{ }_{-2} \psi_{3 \pm 1}^{\text {equator }}}=-\sqrt{\frac{5}{3}}, \quad \frac{{ }_{-2} \psi_{30}^{\text {axis }}}{{ }_{-2} \psi_{33}^{\text {equator }}}=\frac{4}{\sqrt{5}} \text {. }
$$

These proportionality relations are perfectly consistent with the transformation matrices (3.49) and (3.56).

\section{B. Generic rotation angles: unequal-mass "superkick" runs}

As a non-trivial practical application of the transformation laws (3.46) and (3.60b), in this section we study the inspiral and merger of unequal-mass, initially spinning black holes. Our study is a rather striking demonstration of the importance of choosing the "right" reference frame to analyze the radiation produced by a merger event.

Our case-study is the "sk4" run described in Ref. [19]. Our black-hole binary has mass ratio $q \equiv M_{1} / M_{2}=4$. The Kerr parameters of both holes have the same magnitude $\left|j_{1}\right|=\left|j_{2}\right|=0.727$, but opposite directions, and lie on the orbital plane at the beginning of the simulation: this is sometimes referred to as a "superkick" configuration.

Consider the dimensionless spin vector of the remnant black hole $\mathcal{J}=\left(\mathcal{J}_{x}, \mathcal{J}_{y}, \mathcal{J}_{z}\right)$. Define $\mathcal{J}_{x y}=\left(\mathcal{J}_{x}^{2}+\mathcal{J}_{y}^{2}\right)^{1 / 2}$, and let $\mathcal{J}=\left(\mathcal{J}_{x}^{2}+\mathcal{J}_{y}^{2}+\mathcal{J}_{z}^{2}\right)^{1 / 2}$ be the modulus of the final spin. Simple flat-space geometry suggests that a rough but reasonable estimate of the rotation angles relating the simulation frame to the remnant frame should be

$$
\theta_{0}=\arcsin \left(\mathcal{J}_{x y} / \mathcal{J}\right), \quad \phi_{0}=\arccos \left(\mathcal{J}_{x} / \mathcal{J}_{x y}\right) .
$$

For our sk4 run, the final spin can be obtained from angular momentum balance (see Appendix[ Cfor details). The final spin magnitude estimated in this way is $\mathcal{J}=0.642$, and the individual spin components are $\mathcal{J}_{x}=0.424, \mathcal{J}_{y}=0.036$, $\mathcal{J}_{z}=0.480$. The system experiences a significant realignment: the estimated rotation angles are $\theta_{0}=41.5^{\circ}$ and $\phi_{0}=4.8^{\circ}$. Now we can use this estimate of the rotation angles, together with relations (3.46) and (3.60b), to transform the "simulation frame" waveforms to the "remnant frame" waveforms.
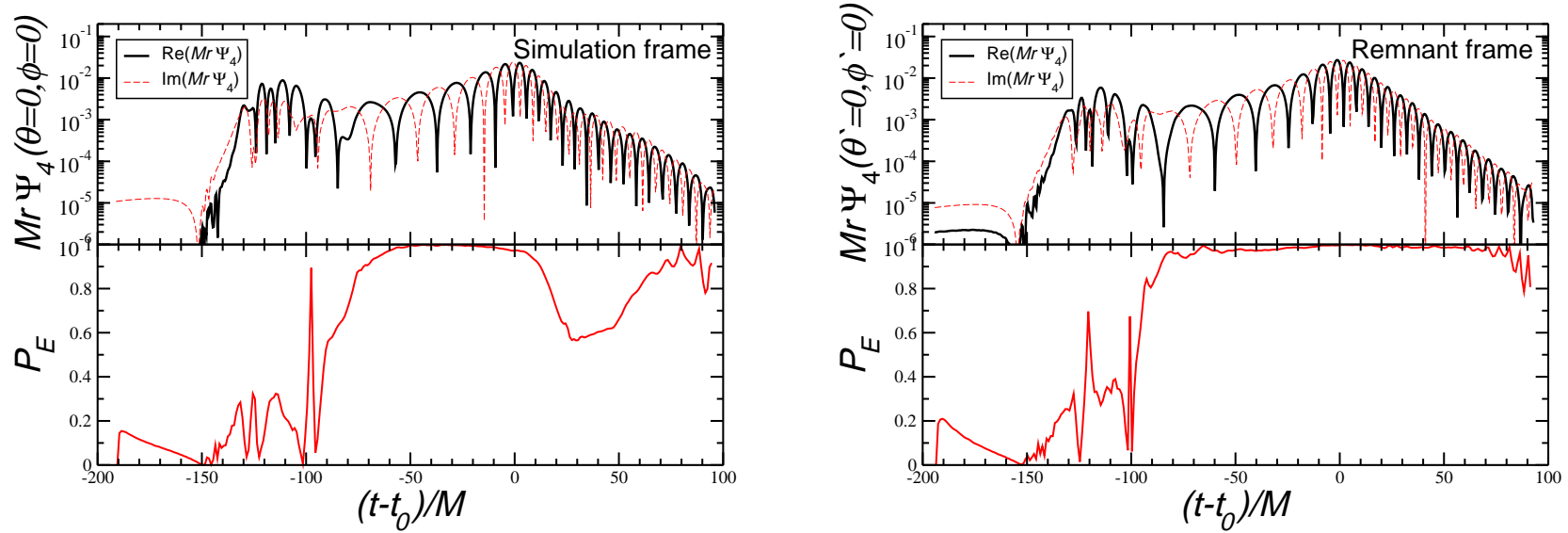

FIG. 2: Top: Quadrupolar $(l=2)$ component of $\left|\operatorname{Re}\left(M r \Psi_{4}\right)\right|$ and $\left|\operatorname{Im}\left(M r \Psi_{4}\right)\right|$ as seen by an observer located on the $z$-axis, for the sk4 run of Ref. [19]. Bottom: degree of elliptic polarization of the waveforms (compare Fig. 28 in Ref. [16]). The left panel refers to the simulation frame, the right panel to the remnant frame.

Our results are summarized in Fig. 2. The upper left panel of Fig. 2 shows the dominant, quadrupolar $(l=2)$ component of $\Psi_{4}$, as seen by an observer located on the $z$-axis in the simulation frame. To obtain this quantity we 
take the sum

$$
\sum_{l=2} \sum_{m=-2,0,2}-2 \psi_{l m}(t, r){ }_{-2} Y_{l m}(\theta, \phi)
$$

of all $l=2$ components with different values of $m$ (i.e. $m=2, m=0$ and $m=-2$ ), and we evaluate it for $\theta=\phi=0$. The upper right panel plots the quadrupolar $(l=2)$ component of $\Psi_{4}$, as seen by an observer on the $z$-axis in the remnant frame. Again, this quantity is obtained by taking the sum of all $l=2$ components in the new frame, as prescribed by the transformation laws for $l=2$. In both plots the time axis is shifted by $t_{0}$, the time corresponding to the peak in the waveform's amplitude, which is also a rough measure for the formation of a common apparent horizon and the beginning of ringdown (see e.g. [16]).

Some simplification in the waveform is clearly seen in the remnant frame, especially in the late (ringdown) phase. In the remnant frame, the ringdown part of the radiation is well described by a single, "clean" damped exponential, and the envelope of the maxima is well fitted by a straight line. In the simulation frame the envelope of the maxima is an oscillating function. This happens because the ringdown radiation is effectively a sum of components with the same $l(=2)$ but different values of $m$, and this produces beatings between different quasinormal modes.

An alternative measure of the improvement produced by using the remnant frame is given by the degree of elliptic polarization $P_{E}$, shown in the bottom panels of Fig. 2. This quantity was defined and discussed in Appendix D of Ref. [16]. It has the property that $P_{E}=1$ for circularly polarized waves, and $P_{E}=0$ for linear polarization. Fig. 2 shows that the ringdown signal is not circularly polarized as seen from the $z$-axis in the simulation frame, but it is indeed circularly polarized (to a very good approximation) in the remnant frame. This is a rather impressive demonstration that the merger-ringdown waveforms look simpler in the remnant frame. On physical grounds this makes perfect sense, since the remnant frame (unlike the simulation frame) is well adapted to the final hole. For generic precessing binaries, it is still possible that switching to the remnant frame does not simplify the early inspiral waveform. A detailed analysis of longer, generic inspiral simulations will be a topic for future research.

It was observed a long time ago that the polarization state of the waves is a useful discriminant between alternative theories of gravity [48, 49]. More recently, it has been suggested that LISA could place interesting bounds on the amplitude birefringence (i.e. the polarization dependent amplification, or attenuation, of the wave amplitude with distance) predicted by Chern-Simons gravity [50]. The fact that the merger-ringdown waveform is circularly polarized in the remnant frame may have useful applications to test general relativity using strong-field observations of merging binaries.

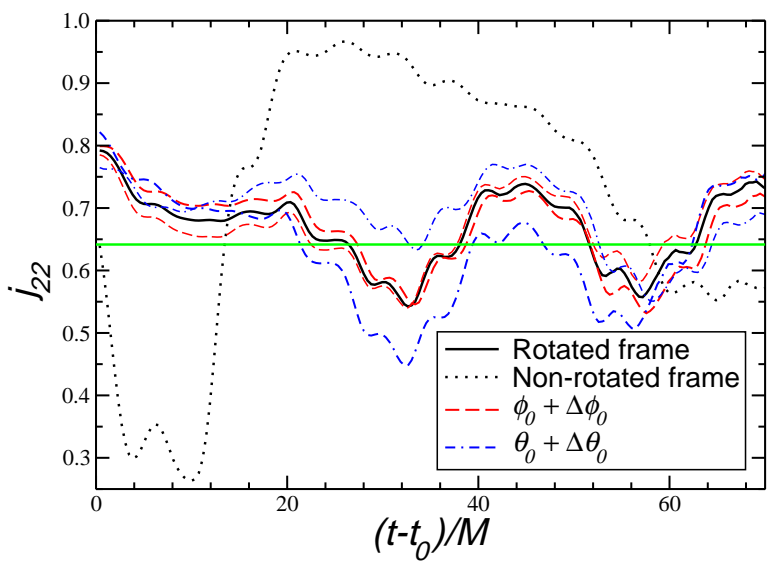

FIG. 3: Final angular momentum from the $(l=2, m=2)$ mode in the remnant frame (solid line) and in the simulation frame (dotted line). Dashed thick (thin) red lines: we arbitrarily increase (decrease) $\phi_{0}$ by $\Delta \phi_{0}=4^{\circ}$. Dash-dash-dotted thick (thin) blue lines: we arbitrarily increase (decrease) $\theta_{0}$ by $\Delta \theta_{0}=8^{\circ}$. The horizontal line represents the estimate of the final spin $(\mathcal{J}=0.642)$ obtained from balance arguments.

As an additional demonstration of the usefulness of the remnant frame it is useful to look at the ringdown radiation. We use a Prony analysis of the $l=m=2$ ringdown mode to extract the quality factor of the quasinormal ringing [32]. From the quality factor we compute the Kerr parameter of the final hole (see [16] and [51] for further details), comparing this estimate with the prediction from angular momentum balance arguments. Our results are shown in 
Fig. 3. where we show the spin estimated by considering different portions of the ringdown signal, starting at time $t>t_{0}$. In the remnant frame, the final spin estimated from the ringdown signal is much closer to the value estimated from balance arguments. The waveform in the simulation frame contains a mixture of various modes of comparable amplitude and different damping times, and this produces a large error in the estimation of the final spin.

As an exercise, in Fig. 3 we also show the final spin estimated in different frames which are "reasonably close" to the remnant frame, considering frames that deviate from our "best guess" for the simulation frame by small angles. For illustration we plot spin estimates obtained assuming $\theta_{0}=41.5^{\circ} \pm \Delta \theta_{0}$ or $\phi_{0}=4.8^{\circ} \pm \Delta \phi_{0}$, where $\Delta \phi_{0}=4^{\circ}$ and $\Delta \theta_{0}=8^{\circ}$. This shows that the good results we obtained in the remnant frame are not accidental: for example, adding (or subtracting) $\Delta \theta_{0}=8^{\circ}$ to our "best estimate" for the rotation angle $\theta_{0}$ seems to produce a systematic underestimate (or overestimate) of the final spin, as obtained from angular momentum balance arguments. Systematic errors in our numerical simulations are admittedly quite large, but the significant bias observed for "incorrect" values of $\Delta \theta_{0}$ is an indication that frames deviating from the remnant frame by small amounts of rotation can yield poor agreement with the final spin estimated from balance arguments.

\section{CONCLUSIONS AND OUTLOOK}

We have provided explicit, ready-to-use formulae to transform multipolar components of gravitational radiation under rotations and non-relativistic boosts. For generic spinning binaries our results indicate that gravitational waveforms are simplest in the "remnant frame" (see Figs. 2] and 3). We provide a practical recipe to identify this frame, and we suggest that all results from multipolar analyses of merger simulations should be expressed in the remnant frame to simplify comparisons. Our work will be useful to standardize file formats [30] and for the injection of numerical waveforms in the data analysis of gravitational-wave detectors [31]. Other interesting applications will be (1) an analysis of the multipolar coupling induced by large boosts in the collision of ultrarelativistic black holes, and (2) a study of the possibility to determine the spin orientation of a binary merger remnant using gravitational-wave observations on Earth and in space. Both topics are currently under investigation.

\section{Acknowledgments}

We are grateful to Curt Cutler, Luis Lehner and Nico Yunes for useful comments on an earlier draft of the manuscript. We also thank the anonymous referee for his constructive criticism. This work was supported in part by DFG grant SFB/Transregio 7 "Gravitational Wave Astronomy". V.C.'s work was partially funded by Fundação para a Ciência e Tecnologia (FCT) - Portugal through projects PTDC/FIS/64175/2006 and POCI/FP/81915/2007. We thank the DEISA Consortium (co-funded by the EU, FP6 project 508830), for support within the DEISA Extreme Computing Initiative (www.deisa.org). Computations were performed at LRZ Munich, the Doppler and Kepler clusters at the Institute of Theoretical Physics of the University of Jena and the Milipeia cluster at the Center for Computational Physics (CFC) in Coimbra. E.B.'s research was supported by an appointment to the NASA Postdoctoral Program at the Jet Propulsion Laboratory, California Institute of Technology, administered by Oak Ridge Associated Universities through a contract with NASA. Government sponsorship acknowledged.

\section{APPENDIX A: BOOSTS AS BMS TRANSFORMATIONS}

Transformations of the form (2.2) and (2.4) are conformal transformations of the $(\theta, \phi)$-sphere into itself, with conformal factor $K$. They correspond to Lorentz transformations of spacetime; indeed, the conformal group $S L(2, C)$ and the Lorentz group $S O(3,1)$ are isomorphic, and any Lorentz transformation on spacetime has, on the sphere, the effect of a conformal transformation (see [2, 3, 21, 36]).

In this Appendix we show this explicitly for the case of a non-relativistic boost with three-velocity $V_{i}$ such that $|V| \ll 1$. We also assume that, since we are interested in asymptotic transformations, $|u| / r \ll 1$. We introduce polar coordinates such that $x_{i}=r n_{i}=r(\sin \theta \cos \phi, \sin \theta \sin \phi, \cos \theta)$, and neglect terms of order $\mathcal{O}\left(V^{2}\right)$ and of order $\mathcal{O}(|u| / r)$. Then under a boost

$$
\begin{aligned}
x_{i}^{\prime} & =x_{i}-V_{i} t, \\
t^{\prime} & =t-V_{i} x_{i} .
\end{aligned}
$$


In polar coordinates we have

$$
\begin{aligned}
r^{\prime} & =\sqrt{x_{i}^{\prime} x_{i}^{\prime}}=r-t V_{i} n_{i} \\
t^{\prime} & =t-r V_{i} n_{i} .
\end{aligned}
$$

It follows that, to linear order in $V$,

$$
u^{\prime}=t^{\prime}-r^{\prime}=u\left(1+V_{i} n_{i}\right)=K u,
$$

with $K(\theta, \phi)=1+V_{i} n_{i}$. This is our main result. Neglecting terms of order $\mathcal{O}(|u| / r)$,

$$
r^{\prime}=r\left(1-\frac{u+r}{r} V_{i} n_{i}\right)=K^{-1} r
$$

The transformation of the director cosines, i.e. of $(\theta, \phi)$, can be found as follows:

$$
x_{i}^{\prime}=r^{\prime} n_{i}^{\prime}=\left(r-t V_{j} n_{j}\right) n_{i}^{\prime}=x_{i}-V_{i} t=r n_{i}-V_{i} t
$$

therefore

$$
n_{i}^{\prime}=\left(1+\frac{t}{r} V_{j} n_{j}\right) n_{i}-\frac{t}{r} V_{i}=\left(1+\frac{u+r}{r} V_{j} n_{j}\right) n_{i}-\frac{u+r}{r} V_{i}=\left(1+V_{j} n_{j}\right) n_{i}-V_{i}=K n_{i}-V_{i}
$$

These are well-defined director cosines: indeed, to linear order in $V$,

$$
n_{i}^{\prime} n_{i}^{\prime}=\left(K n_{i}-V_{i}\right)\left(K n_{i}-V_{i}\right)=K^{2}-2 n_{i} V_{i}=1 .
$$

Using the equality $2 K n_{i} V_{i}=2\left(1+V_{i} n_{i}\right) n_{i} V_{i}=2 n_{i} V_{i}+\mathcal{O}\left(V^{2}\right)$ and consistently neglecting terms of $\mathcal{O}\left(V^{2}\right)$, it can be shown that the metric on the two-sphere changes as follows:

$$
d \theta^{\prime 2}+\sin ^{2} \theta^{\prime} d \phi^{\prime 2}=d n_{i}^{\prime} d n_{i}^{\prime}=K^{2} d n_{i} d n_{i}=K^{2}\left(d \theta^{2}+\sin ^{2} \theta d \phi^{2}\right) .
$$

The boosts, then, act on $(u, \theta, \phi)$ as in Eqs. (2.2) and (2.4). Notice that the flat spacetime metric does not change under a boost, i.e.

$$
d s^{2}=-d u^{2}-d u d r+r^{2} d n_{i} d n_{i}=-d u^{\prime 2}-2 d u^{\prime} d r^{\prime}+r^{2} d n_{i}^{\prime} d n_{i}^{\prime}
$$

but this is not obvious by replacing (A3), (A4) and (A6) in the above relation. The reason is that we have neglected terms with $u / r \ll 1$. As a qualitative check of the expression $K=1+V_{i} n_{i}$, let us consider the case in which the observers are boosted towards the source. Then $V_{i} n_{i}<0$, and $K=1+V_{i} n_{i}<1$. Therefore, the observed gravitational-wave frequency changes as $\omega^{\prime}=\omega / K>\omega$, i.e. we see a blueshifted wave, as expected.

We have shown that a boost with velocity $\vec{V}$ such that $|V| \ll 1$ has conformal factor $K=1+V_{i} n_{i}$. It affects the transformation of the asymptotic Weyl scalar $\Psi_{4}$, Eq. (3.21), by terms linear in $V$. We also have to consider the effect of the change in the coordinates, which is of order $V$ as can be seen from (A3), (A4) and (A6), and is harder to derive explicitly. It is derived in a particular case, in Section IIIC2. Notice that, if the black hole kick determines both a boost and a translation, the translation only appears in the change of coordinates, and not in the transformation (3.21). A translation is expected to give a contribution of order $|\delta \vec{x}| / r$, which is extremely small far away from the source: for the purpose of detection, the boost is the most relevant transformation.

\section{APPENDIX B: THE STEREOGRAPHIC REPRESENTATION}

Conformal transformations of the sphere (which correspond to asymptotic Lorentz transformations of spacetime) can be expressed as conformal transformations on the complex plane obtained by projecting the sphere from the north pole to the equatorial plane $\left(y_{1}, y_{2}\right)$. From the geometry in Fig. 4 , defining a complex variable $\zeta=y_{1}+\mathrm{i} y_{2}$ we have

$$
\zeta=\cot \frac{\theta}{2} e^{i \phi}
$$

The 2-sphere metric can be written as

$$
d \theta^{2}+\sin ^{2} \theta d \phi^{2}=4(1+\zeta \bar{\zeta})^{-2} d \zeta d \bar{\zeta}
$$




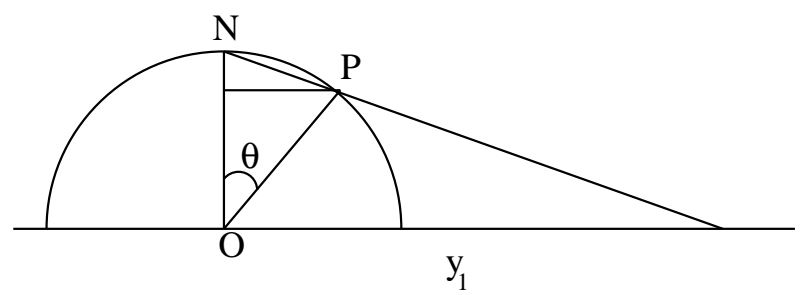

FIG. 4: Stereographic projection of a sphere onto the plane.

The director $\operatorname{cosines} n_{i}=(\sin \theta \cos \phi, \sin \theta \sin \phi, \cos \theta)$ are related to $\zeta$ by

$$
n_{1}+\mathrm{i} n_{2}=\frac{2 \zeta}{\zeta \bar{\zeta}+1}, \quad n_{3}=\frac{\zeta \bar{\zeta}-1}{\zeta \bar{\zeta}+1}
$$

and by the inverse relation, $\zeta=\left(n_{1}+\mathrm{i} n_{2}\right) /\left(1-n_{3}\right)$. An $S L(2, C)$ transformation

$$
A=\left(\begin{array}{ll}
a & b \\
c & d
\end{array}\right) \text {, }
$$

maps $\zeta$ to $\zeta^{\prime}$ as in Eq. (2.6), with $a d-b c=1$. Notice that if $a d-b c \neq 0$ we can rescale the four elements without changing the transformation, in order to have $a d-b c=1$. This can be shown to be a conformal transformation, i.e. the 2-sphere metric changes as in Eq. (2.2), which in stereographic coordinates means

$$
4\left(1+\zeta^{\prime} \bar{\zeta}^{\prime}\right)^{-2} d \zeta^{\prime} d \bar{\zeta}^{\prime}=K^{2} 4(1+\zeta \bar{\zeta})^{-2} d \zeta d \bar{\zeta}
$$

A straightforward calculation shows that

$$
K^{-1}=\frac{(a \zeta+c)(\bar{a} \bar{\zeta}+\bar{c})+(b \zeta+d)(\bar{b} \bar{\zeta}+\bar{d})}{\zeta \bar{\zeta}+1} .
$$

The phase $\chi$ appearing in Eqs. (3.15) and (3.17) is given by (see Eq. (3.12) in [2])

$$
e^{-2 \mathrm{i} \chi}=\frac{\partial \zeta^{\prime} / \partial \zeta}{\partial \bar{\zeta}^{\prime} / \partial \bar{\zeta}}=\frac{(\bar{b} \bar{\zeta}+\bar{d})^{2}}{(b \zeta+d)^{2}}
$$

\section{Rotations in stereographic coordinates}

The main purpose of this Section is to derive the specialization of (2.6) to a rotation by $\left(\theta_{0}, \phi_{0}\right)$, i.e. Eq. (3.44), and its special case Eq. (3.47) for rotations by an angle $\theta_{0}=\pi / 2$. Rotations are $S L(2, C)$ transformations for which $K=1$. Using Eq. (B6) this implies

$$
(a \zeta+c)(\bar{a} \bar{\zeta}+\bar{c})+(b \zeta+d)(\bar{b} \bar{\zeta}+\bar{d})=\zeta \bar{\zeta}+1
$$

and therefore

$$
\begin{aligned}
|a|^{2}+|b|^{2} & =1, \\
|c|^{2}+|d|^{2} & =1, \\
a \bar{c}+b \bar{d} & =0 .
\end{aligned}
$$

But $a d-b c=1$, so $A \in S U(2)$ and

$$
a=\bar{d}, \quad b=-\bar{c} .
$$

Let us first consider, for simplicity, a rotation of $\pi / 2$ around the $x_{2}$-axis; it maps $x_{2} \rightarrow x_{2}, x_{1} \rightarrow x_{3}$ and $x_{3} \rightarrow-x_{1}$, i.e.

$$
\begin{aligned}
\sin \theta^{\prime} \cos \phi^{\prime} & =-\cos \theta, \\
\sin \theta^{\prime} \sin \phi^{\prime} & =\sin \theta \sin \phi, \\
\cos \theta^{\prime} & =\sin \theta \cos \phi .
\end{aligned}
$$


In particular, the plane $\phi=\phi^{\prime}=0$ is left unchanged, and $\theta^{\prime}=\theta-\frac{\pi}{2}$. The corresponding function $\zeta^{\prime}(\zeta)$ maps (in the plane $\phi=0)-1$ in 0,0 in 1 and 1 in $\infty$, therefore it must have the form

$$
\zeta^{\prime}=\frac{a \zeta+c}{b \zeta+d}=\frac{\alpha(\zeta+1)}{\alpha(-\zeta+1)}
$$

and imposing $a d-b c=1$ this gives Eq. (3.47). As expected, this transformation satisfies the relations (B9), thus $K=1$.

By appropriately choosing the primed frame, a general rotation maps a vector with generic orientation to the $z^{\prime}$-axis:

$$
\left(J_{x}, J_{y}, J_{z}\right)=\left(J \sin \theta_{0} \cos \phi_{0}, J \sin \theta_{0} \sin \phi_{0}, J \cos \theta_{0}\right) \rightarrow(0,0, J) .
$$

Therefore, it maps $\zeta_{0}=\cot \left(\theta_{0} / 2\right) e^{\mathrm{i} \phi_{0} / 2}$ to $\zeta^{\prime}=\infty$. Imposing (B9) (i.e. $K=1$ ) yields

$$
\zeta^{\prime}=\frac{\cos \frac{\theta_{0}}{2} e^{-\mathrm{i} \frac{\phi_{0}}{2}} \zeta+\sin \frac{\theta_{0}}{2} e^{\mathrm{i} \frac{\phi_{0}}{2}}}{-\sin \frac{\theta_{0}}{2} e^{-\mathrm{i} \frac{\phi_{0}}{2}}+\cos \frac{\theta_{0}}{2} e^{\mathrm{i} \frac{\phi_{0}}{2}}}
$$

i.e. Eq. (3.44).

\section{Boosts in stereographic coordinates}

Let us now express boosts in stereographic coordinates. As shown in Appendix A. the conformal factor of a boost with velocity $V_{i}$ such that $|V| \ll 1$ is $K=1+V_{i} n_{i}$. We define

$$
V_{ \pm}=V_{1} \pm \mathrm{i} V_{2}
$$

thus

$$
V_{i} n_{i}=V_{+} \frac{n_{1}-\mathrm{i} n_{2}}{2}+V_{-} \frac{n_{1}+\mathrm{i} n_{2}}{2}+V_{3} n_{3}=\frac{1}{\zeta \bar{\zeta}+1}\left[V_{+} \bar{\zeta}+V_{-} \zeta+V_{3}(\zeta \bar{\zeta}-1)\right]
$$

Therefore, to linear order in $V$,

$$
\begin{aligned}
K & =1+\frac{1}{\zeta \bar{\zeta}+1}\left[V_{+} \bar{\zeta}+V_{-} \zeta+V_{3}(\zeta \bar{\zeta}-1)\right] \\
K^{-1} & =1-\frac{1}{\zeta \bar{\zeta}+1}\left[V_{+} \bar{\zeta}+V_{-} \zeta+V_{3}(\zeta \bar{\zeta}-1)\right]
\end{aligned}
$$

Now we will work out the expression of a boost as an $S L(2, C)$ transformation. A four-vector $x^{\mu}$ in spacetime can be written as an $S L(2, C)$ matrix $X$ :

$$
X=\sigma_{\mu} x_{\mu}=\left(\begin{array}{cc}
x_{0}+x_{3} & x_{1}-\mathrm{i} x_{2} \\
x_{1}+\mathrm{i} x_{2} & x_{0}-x_{3}
\end{array}\right)
$$

where $\sigma_{0}$ is the identity matrix and $\sigma_{i}(i=1,2,3)$ are the ordinary Pauli matrices. A boost in the $x_{1}$ direction is represented by the matrix

$$
A=e^{-\frac{\sigma_{1} \omega}{2}}=\cosh \frac{\omega}{2}-\sigma_{1} \sinh \frac{\omega}{2}=\left(\begin{array}{cc}
\cosh \frac{\omega}{2} & -\sinh \frac{\omega}{2} \\
-\sinh \frac{\omega}{2} & \cosh \frac{\omega}{2}
\end{array}\right)
$$

where the velocity of the boost is $V=\tanh \omega$. Indeed, the transformed vector is

$$
A X A^{\dagger}=\left(\begin{array}{cc}
x_{0} \cosh \omega-x_{1} \sinh \omega+x_{3} & -x_{0} \sinh \omega+x_{1} \cosh \omega-\mathrm{i} x_{2} \\
-x_{0} \sinh \omega+x_{1} \cosh \omega+\mathrm{i} x_{2} & x_{0} \cosh \omega-x_{1} \sinh \omega-x_{3}
\end{array}\right) .
$$

where a dagger denotes Hermitian conjugation. For a boost in a generic direction, defining three parameters $\omega_{i}$ $(i=1,2,3)$ we have

$$
A=e^{-\frac{\sigma_{i} \omega_{i}}{2}}=\cosh \frac{\omega_{i}}{2}-\sigma_{i} \sinh \frac{\omega_{i}}{2}
$$


To linear order $\omega=V+\mathcal{O}\left(V^{2}\right)$, and

$$
A \simeq 1-\sigma_{i} \frac{V_{i}}{2}=\left(\begin{array}{cc}
1-\frac{V_{3}}{2} & -\frac{V_{-}}{2} \\
-\frac{V_{+}}{2} & 1+\frac{V_{3}}{2}
\end{array}\right)=\left(\begin{array}{ll}
a & b \\
c & d
\end{array}\right)
$$

which is the result quoted in Eq. (3.57). Such a boost maps $\zeta=\cot \frac{\theta}{2} e^{\mathrm{i} \phi}$ into

$$
\zeta^{\prime}=\frac{a \zeta+c}{b \zeta+d}=\frac{\left(1-\frac{V_{3}}{2}\right) \zeta-\frac{V_{+}}{2}}{1+\frac{V_{3}}{2}-\frac{V_{-}}{2} \zeta} \simeq \zeta\left(1-\frac{V_{3}}{2}+\frac{V_{-}}{2}\right)-\frac{V_{3}}{2}-\frac{V_{-}}{2},
$$

and

$$
e^{\mathrm{i} \chi}=\frac{b \zeta+d}{\bar{b} \bar{\zeta}+\bar{d}}=\frac{1+\frac{V_{3}}{2}-\frac{V_{-} \zeta}{2}}{1+\frac{V_{3}}{2}-\frac{V_{+} \bar{\zeta}}{2}} \simeq 1+\frac{V_{+} \bar{\zeta}+V_{-} \zeta}{2}=1+\mathrm{i} \cot \frac{\theta}{2}\left[V_{2} \cos \phi-V_{1} \sin \phi\right] .
$$

As a check of Eq. (3.57) it is easy to verify that the inverse conformal factor (B6), when expanded to linear order in $V$, agrees with Eq. (B18).

\section{APPENDIX C: CALCULATION OF THE RADIATED ANGULAR MOMENTUM IN NUMERICAL EVOLUTIONS OF BLACK-HOLE BINARIES}

All numerical black-hole binary evolutions in this paper have been performed using the LEAN code [43]. We compute the energy, linear and angular momentum radiated during merger using the Newman-Penrose scalar $\Psi_{4}$. The calculation of $\Psi_{4}$, as implemented in the LEAN code, is described in detail in Appendix C of Ref. [43]. Here we assume $\Psi_{4}$ as given on an extraction sphere of constant coordinate radius $r_{\mathrm{ex}}$.

The radiated energy, the $(x, y, z)$ components of the radiated linear momentum and the $z$ component of the radiated angular momentum are then given, for example, by Eqs. (22)-(24) of Ref. [52]. For the simplest binary black hole configurations these quantities encapsulate the entire information about the radiated momenta, because the $x$ and $y$ components of $J_{\text {rad }}$ are known to vanish from symmetry arguments. However, for more general classes of spinning binaries (such as those considered in this paper) we can no longer neglect these components. The calculation of $J_{x, \text { rad }}$ and $J_{y, \text { rad }}$ from $\Psi_{4}$ has recently been discussed in [53, 54]. Here we follow Eqs. $(2.24,2.25)$ of [54] and calculate the radiated angular momentum from

$$
\frac{d J_{i, \mathrm{rad}}}{d t}=-\lim _{r \rightarrow \infty} \frac{r^{2}}{16 \pi} \operatorname{Re} \oint \hat{J}_{i}\left[\int_{-\infty}^{t}\left(\int_{-\infty}^{\tilde{t}} \Psi_{4} d \bar{t}\right) d \tilde{t}\right]\left(\int_{-\infty}^{t} \Psi_{4} d \tilde{t}\right) d \Omega
$$

where

$$
\begin{aligned}
\sin \theta \hat{J}_{x} & =-\sin \theta \sin \phi \partial_{\theta}-\cos \theta \cos \phi \partial_{\phi}+i s \cos \phi \\
\sin \theta \hat{J}_{y} & =\sin \theta \cos \phi \partial_{\theta}-\cos \theta \sin \phi \partial_{\phi}+i s \cos \phi \\
\hat{J}_{z} & =\partial_{\phi}
\end{aligned}
$$

and the spin weight $s=-2$.

As a test of the numerical implementation of these expressions we followed the suggestion of Sec. III of Ref. [53]. We evolved the binary configuration labelled "R1" in table I of Ref. [55] three times, the orbital angular momentum $L$ being aligned with the $z, y$ and $x$ axis, but always using the $z$ axis as reference for the wave extraction. We use the grid setup of Table I in [43] adapted to the orientation of $L$, using a comparatively low resolution $h=1 / 32$ for these test simulations. The resulting radiated angular momentum is shown in Fig. 5. The radiated angular momenta in the three simulations agree within a few percent, which for these low resolution runs is less than the uncertainties resulting from the discretization.

\section{APPENDIX D: RADIAL INFALL OF PARTICLES INTO NON-ROTATING BLACK HOLES}

Small perturbations of the Kerr geometry are usually described using the Teukolsky formalism 41, 56., but some practical applications require a modification of the equations. The Sasaki-Nakamura formalism [57, 58, 59, 60, 61, 


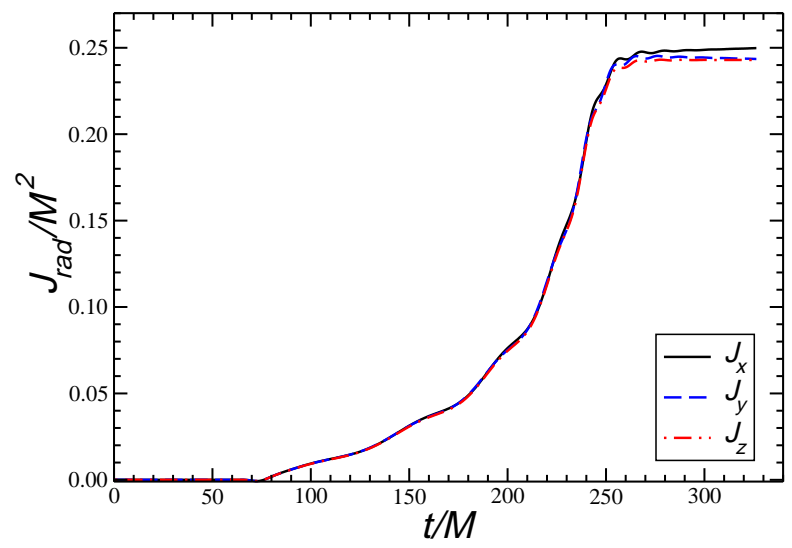

FIG. 5: Radiated angular momentum as a function of time from the R1 simulation with the orbital angular momentum aligned with the $x, y$ and $z$ axis (see text).

62, 63, 64, 65] is one such modification that is very useful (among other applications) to deal with the infall of point particles into Kerr black holes. For simplicity, here we consider a highly relativistic point particle falling into a non-rotating black hole, since in this case the equations simplify considerably [66, 67]. Our purpose is to check the formalism developed in the text by considering the same problem in two different reference frames. In the first case, we consider the radial infall of a particle along the $z$-axis, making full use of the symmetries of the problem. In the second case, we deliberately let the particle fall in along a radial trajectory on the equatorial plane, destroying the symmetry of the problem. The point of this Appendix is to derive the source terms of the Sasaki-Nakamura equation in these two configurations; using such expression, in Section IVA2 we show that the transformation properties derived in this paper are perfectly compatible with the transformation laws of the source term of the Sasaki-Nakamura equation.

Details about the Teukolsky formalism may be found in the original literature [41, 56], and also in [68]. Working in the Kinnersley tetrad, the equations for the Newman-Penrose quantities decouple and separate, giving rise to the Teukolsky equation for a radial function $R[41,56]$ :

$$
\Delta^{-s} \frac{d}{d r}\left(\Delta^{s+1} \frac{d}{d r} R\right)-{ }_{s} V R=-T
$$

where we defined $\Delta \equiv r^{2}-2 M r$, and $M$ is the mass of the black hole. The quantity $T$ is a source term due to the stress-energy of the particle, and the potential

$$
{ }_{s} V=\frac{-K^{2}}{\Delta}+\frac{\mathrm{i} s K \Delta^{\prime}}{\Delta}-\frac{2 \mathrm{i} s K^{\prime}}{\Delta}-2 \mathrm{i} s K^{\prime}+{ }_{s} \lambda .
$$

We work in the frequency domain, assuming a time dependence of the form $e^{-\mathrm{i} \omega t}$. The function $K \equiv r^{2} \omega$, and primes denote derivatives with respect to $r$. The quantity $s$ is the spin weight (or helicity) of the field under consideration (see for example [39]), and $m$ is an azimuthal quantum number. We are interested in gravitational perturbations, which have spin-weight $s=-2$. For non-rotating black holes the separation constant ${ }_{s} \lambda=l(l+1)-s(s+1)$. The source term $T$ appearing in Eq. (D1) depends on the stress-energy tensor of the perturbation under consideration (see e.g. [68]). For a test particle falling in from infinity with zero velocity $T \sim r^{7 / 2}$, and the potential ${ }_{s} V$ is always long-ranged. This implies that when we try to solve the Teukolsky equation (D1) numerically we will run into problems. Usually the numerical solution is accomplished by integrating the source term multiplied by certain homogeneous solutions. At infinity the integral will not be well-defined: the source term blows up there, and because the potential is long range, the homogeneous solution will also blow up at infinity. We can remedy this long-range nature of the potential, and at the same time regularize the source term, by using the Sasaki-Nakamura formalism. After some manipulation, the Teukolsky equation (D1) may be cast in the Sasaki-Nakamura form [57, 58, 59, 60, 61, 62, 63]:

$$
\frac{d^{2}}{d r_{*}^{2}} X(\omega, r)-\mathcal{F} \frac{d}{d r_{*}} X(\omega, r)-\mathcal{U} X(\omega, r)=\mathcal{L} .
$$


The functions $\mathcal{F}$ and $\mathcal{U}$ can be found in the original literature [57, 58, 59, 60, 61, 62, 63]. The source term $\mathcal{L}$ is given by

$$
\mathcal{L}=\frac{\gamma_{0} \Delta}{r^{5}} W e^{-\mathrm{i} \int K / \Delta d r_{*}}
$$

and $W$ satisfies

$$
W^{\prime \prime}=-\frac{r^{2}}{\Delta} T \exp \left[\mathrm{i} \int \frac{K}{\Delta} d r_{*}\right]
$$

The tortoise coordinate $r_{*}$, defined by $d r_{*} / d r \equiv r^{2} / \Delta$, ranges from $-\infty$ at the horizon to $+\infty$ at spatial infinity. The Sasaki-Nakamura equation (D3) must be solved imposing an outgoing-radiation boundary condition at infinity:

$$
X(\omega, r)=X^{\text {out }} e^{\mathrm{i} \omega r_{*}}, \quad r_{*} \rightarrow \infty
$$

The two independent polarization modes of the metric, $h_{+}$and $h_{\times}$, are then given by

$$
h_{+}+\mathrm{i} h_{\times}=\frac{8}{r} \int_{-\infty}^{+\infty} d \omega \sum_{l, m} e^{\mathrm{i} \omega\left(r_{*}-t\right)} \frac{X^{\text {out }}-2 Y_{l m}(\theta, \phi)}{\lambda(\lambda+2)-12 \mathrm{i} \omega} .
$$

Using a formal Green's function solution it is easy to see that $X^{\text {out }} \propto \mathcal{L}$.

Let us now consider the radial infall of an ultrarelativistic point particle into a non-rotating black hole. The dependence of the emitted gravitational wave power on the trajectory of the particle comes entirely from the source $\mathcal{L}$ in eqs. (D3)-(D4), which in turn depends on the source term $T$ in Teukolsky's equation (D1) through Eq. (D5). To compute the explicit dependence, one would have to consider generic geodesics in the Kerr geometry [69], which in general gives an end result not very amenable to analytical treatments. However in the highly relativistic regime the equations simplify enormously [66, 67], and in particular for radial infall (along the symmetry axis or along the equator) it is possible to find a simple closed-form analytic expression for the function $W$ in Eq. (D4). Accordingly, in the following we will provide a closed-form expressions for $\mathcal{L}$ in two special situations: radial infall of an ultrarelativistic particle along the symmetry axis, and radial infall of an ultrarelativistic particle on the equatorial plane. We will further specialize to non-rotating black holes, where analytic expressions for the spin-weighted harmonics are known.

\section{Particle falling along the symmetry axis}

Consider a particle falling radially along the symmetry axis of a Kerr black hole. In this case the geodesics, written in Boyer-Lindquist coordinates, are

$$
\frac{d t}{d \tau}=\epsilon_{0} \frac{r^{2}}{\Delta}, \quad\left(\frac{d r}{d \tau}\right)^{2}=\epsilon_{0}^{2}-\frac{\Delta}{r^{2}}, \quad \frac{d \phi}{d \tau}=0,
$$

where the parameter $\epsilon_{0}$ is the energy per unit rest mass of the infalling particle. On considering highly relativistic particles, $\epsilon_{0} \rightarrow \infty$, the source-term function $\mathcal{L}$ reduces to [66, 67]

$$
\mathcal{L}=-\frac{\mu C \epsilon_{0} \gamma_{0} \Delta}{2 \omega^{2} r^{5}} e^{-i \omega r_{*}}
$$

Here $C=8\left(-2 Y_{l 0} / \sin ^{2} \theta\right)_{\theta=0}$, the function $\gamma_{0}=\gamma_{0}(r)$ can be found in [57, 58, 59, 60, 61, 62, 63], and $\mu$ is the mass of the particle. We find

$$
\begin{aligned}
\mathcal{L}^{\text {axis }} & =-\frac{\mu \sqrt{\frac{15}{2 \pi}} \epsilon_{0} \gamma_{0} \Delta}{\omega^{2} r^{5}} e^{-\mathrm{i} \omega r_{*}}, \quad(l=2), \\
\mathcal{L}^{\text {axis }} & =-\frac{\mu \sqrt{\frac{105}{2 \pi}} \epsilon_{0} \gamma_{0} \Delta}{\omega^{2} r^{5}} e^{-\mathrm{i} \omega r_{*}}, \quad(l=3) .
\end{aligned}
$$




\section{Particle falling on the equatorial plane}

Now suppose that the particle is falling radially on the equatorial plane of a Kerr black hole. On considering highly relativistic particles we find that the source term takes again a very simple form [66, 67]:

$$
\mathcal{L}=-\frac{\mu \hat{S} \epsilon_{0} \gamma_{0} \Delta}{\omega^{2} r^{5}} e^{-\mathrm{i} \int \frac{K}{\Delta} d r}
$$

where

$$
\hat{S}=\left[\frac{\lambda}{2}-m^{2}\right]{ }_{-2} Y_{l m}(\pi / 2,0)+m_{-2} Y_{l m}^{\prime}(\pi / 2,0),
$$

and a prime stands for derivative with respect to $\theta$. For the dominant multipolar components we get

$$
\begin{aligned}
& \mathcal{L}_{20}^{\text {equator }}=-\frac{\mu \sqrt{\frac{15}{2 \pi}} \epsilon_{0} \gamma_{0} \Delta}{2 \omega^{2} r^{5}} e^{-\mathrm{i} \omega r_{*}}, \quad \mathcal{L}_{2 \pm 2}^{\text {equator }}=\frac{3 \mu \sqrt{\frac{5}{\pi}} \epsilon_{0} \gamma_{0} \Delta}{4 \omega^{2} r^{5}} e^{-\mathrm{i} \omega r_{*}}, \\
& \mathcal{L}_{3 \pm 3}^{\text {equator }}= \pm \frac{5 \mu \sqrt{\frac{21}{2 \pi}} \epsilon_{0} \gamma_{0} \Delta}{4 \omega^{2} r^{5}} e^{-\mathrm{i} \omega r_{*}}, \quad \mathcal{L}_{3 \pm 1}^{\text {equator }}=\mp \frac{3 \mu \sqrt{\frac{35}{2 \pi}} \epsilon_{0} \gamma_{0} \Delta}{4 \omega^{2} r^{5}} e^{-\mathrm{i} \omega r_{*}} .
\end{aligned}
$$

[1] K. S. Thorne, Rev. Mod. Phys. 52, 299 (1980).

[2] E. T. Newman and R. Penrose, J. Math. Phys. 7, 863 (1966).

[3] J. N. Goldberg, A. J. MacFarlane, E. T. Newman, F. Rohrlich, and E. C. G. Sudarshan, J. Math. Phys. 8, 2155 (1967).

[4] J. G. Baker, J. R. van Meter, S. T. McWilliams, J. Centrella, and B. J. Kelly, Phys. Rev. Lett. 99, 181101 (2007), gr-qc/0612024.

[5] Y. Pan et al., Phys. Rev. D77, 024014 (2008), arXiv:0704.1964 [gr-qc].

[6] A. Buonanno et al., Phys. Rev. D76, 104049 (2007), arXiv:0706.3732 [gr-qc].

[7] M. Boyle et al., Phys. Rev. D76, 124038 (2007), arXiv:0710.0158 [gr-qc].

[8] M. Hannam, S. Husa, U. Sperhake, B. Brugmann, and J. A. Gonzalez, Phys. Rev. D77, 044020 (2008), arXiv:0706.1305 [gr-qc].

[9] T. Damour and A. Nagar, Phys. Rev. D77, 024043 (2008), arXiv:0711.2628 [gr-qc].

[10] P. Ajith et al., Class. Quant. Grav. 24, S689 (2007), arXiv:0704.3764 [gr-qc].

[11] P. Ajith et al. (2007), arXiv:0710.2335 [gr-qc].

[12] N. Yunes and E. Berti (2008), arXiv:0803.1853 [gr-qc].

[13] L. E. Kidder, Phys. Rev. D77, 044016 (2008), arXiv:0710.0614 [gr-qc].

[14] L. Blanchet, G. Faye, B. R. Iyer, and S. Sinha (2008), arXiv:0802.1249 [gr-qc].

[15] A. Buonanno, G. B. Cook, and F. Pretorius, Phys. Rev. D75, 124018 (2007), gr-qc/0610122.

[16] E. Berti et al., Phys. Rev. D76, 064034 (2007), gr-qc/0703053.

[17] J. D. Schnittman et al., Phys. Rev. D77, 044031 (2008), arXiv:0707.0301 [gr-qc].

[18] B. Vaishnav, I. Hinder, F. Herrmann, and D. Shoemaker, Phys. Rev. D76, 084020 (2007), arXiv:0705.3829 [gr-qc].

[19] E. Berti, V. Cardoso, J. A. Gonzalez, U. Sperhake, and B. Brugmann (2007), arXiv:0711.1097 [gr-qc].

[20] H. Bondi, M. G. J. van der Burg, and A. W. K. Metzner, Proc. Roy. Soc. Lond. A269, 21 (1962).

[21] R. Sachs, Phys. Rev. 128, 2851 (1962).

[22] R. K. Sachs, Proc. Roy. Soc. Lond. A270, 103 (1962).

[23] M. Campanelli, C. O. Lousto, Y. Zlochower, and D. Merritt, Astrophys. J. 659, L5 (2007), gr-qc/0701164.

[24] M. Campanelli, C. O. Lousto, Y. Zlochower, and D. Merritt, Phys. Rev. Lett. 98, 231102 (2007), gr-qc/0702133.

[25] J. A. Gonzalez, M. D. Hannam, U. Sperhake, B. Brugmann, and S. Husa, Phys. Rev. Lett. 98, 231101 (2007), grqc/0702052.

[26] D. Pollney et al., Phys. Rev. D76, 124002 (2007).

[27] S. Dain, C. O. Lousto, and Y. Zlochower (2008), arXiv:0803.0351 [gr-qc].

[28] E. E. Flanagan and S. A. Hughes, Phys. Rev. D57, 4535 (1998), gr-qc/9701039.

[29] E. Berti, J. Cardoso, V. Cardoso, and M. Cavaglia, Phys. Rev. D76, 104044 (2007), arXiv:0707.1202 [gr-qc].

[30] D. Brown et al. (2007), arXiv:0709.0093 [gr-qc].

[31] http://www.gravity.phy.syr.edu/dokuwiki/doku.php?id=ninja:home

[32] E. Berti, V. Cardoso, and C. M. Will, Phys. Rev. D73, 064030 (2006), gr-qc/0512160.

[33] U. Sperhake, V. Cardoso, F. Pretorius, E. Berti, and J. A. Gonzalez (2008), arXiv:0806.1738 [gr-qc].

[34] P. J. McCarthy, Journal of Mathematical Physics 13, 1837 (1972).

[35] R. Wald, General Relativity (The University of Chicago press, Chicago, 1984). 
[36] M. Carmeli, Group theory and general relativity: Representations of the Lorentz group and their applications to the gravitational field (World Scientific, Singapore, 2000).

[37] E. T. Newman and T. W. J. Unti, Journal of Mathematical Physics 3, 891 (1962).

[38] R. Geroch, in Asymptotic Structure of Space-Time, edited by F. P. Esposito and L. Witten (Plenum Press, New York, 1977).

[39] E. Newman and R. Penrose, Journal of Mathematical Physics 3, 566 (1962).

[40] L. Lehner and O. M. Moreschi, Phys. Rev. D76, 124040 (2007), arXiv:0706.1319 [gr-qc].

[41] S. A. Teukolsky, Astrophys. J. 185, 635 (1973).

[42] B. Brugmann et al., Phys. Rev. D77, 024027 (2008), gr-qc/0610128.

[43] U. Sperhake, Phys. Rev. D76, 104015 (2007), gr-qc/0606079.

[44] C. W. Misner, K. Thorne, and J. A. Wheeler, Gravitation (W. H. Freeman \& Co., San Francisco, 1973).

[45] J. D. Brown, S. R. Lau, and J. York, James W., Phys. Rev. D55, 1977 (1997), gr-qc/9609057.

[46] U. Sperhake et al. (2007), arXiv:0710.3823 [gr-qc].

[47] C. Palenzuela, I. Olabarrieta, L. Lehner, and S. Liebling, Phys. Rev. D75, 064005 (2007), gr-qc/0612067.

[48] D. M. Eardley, D. L. Lee, A. P. Lightman, R. V. Wagoner, and C. M. Will, Phys. Rev. Lett. 30, 884 (1973).

[49] D. M. Eardley, D. L. Lee, and A. P. Lightman, Phys. Rev. D8, 3308 (1973).

[50] S. Alexander, L. S. Finn, and N. Yunes (2007), arXiv:0712.2542 [gr-qc].

[51] E. Berti, V. Cardoso, J. A. Gonzalez, and U. Sperhake, Phys. Rev. D75, 124017 (2007), arXiv:0701086 [gr-qc].

[52] M. Campanelli and C. O. Lousto, Phys. Rev. D59, 124022 (1999), gr-qc/9811019.

[53] C. O. Lousto and Y. Zlochower, Phys. Rev. D76, 041502 (2007), gr-qc/0703061.

[54] M. Ruiz, R. Takahashi, M. Alcubierre, and D. Nunez (2007), arXiv:0707.4654 [gr-qc].

[55] J. G. Baker, J. Centrella, D.-I. Choi, M. Koppitz, and J. van Meter, Phys. Rev. D73, 104002 (2006), gr-qc/0602026.

[56] S. A. Teukolsky, Phys. Rev. Lett. 29, 1114 (1972).

[57] M. Sasaki and T. Nakamura, Prog. Theor. Phys. 67, 1788 (1982).

[58] T. Nakamura and M. Sasaki, Phys. Lett. A89, 185 (1982).

[59] M. Sasaki and T. Nakamura, Phys. Lett. A89, 68 (1982).

[60] Y. Kojima and T. Nakamura, Progress of Theoretical Physics 71, 79 (1984).

[61] Y. Kojima and T. Nakamura, Physics Letters A 96, 335 (1983).

[62] Y. Kojima and T. Nakamura, Physics Letters A 99, 37 (1983).

[63] M. Sasaki and T. Nakamura, Physics Letters A 87, 85 (1981).

[64] S. A. Hughes, Phys. Rev. D61, 084004 (2000), gr-qc/9910091.

[65] D. Kennefick, Phys. Rev. D58, 064012 (1998), gr-qc/9805102.

[66] V. Cardoso and J. P. S. Lemos, Phys. Lett. B538, 1 (2002), gr-qc/0202019.

[67] V. Cardoso and J. P. S. Lemos, Phys. Rev. D67, 084005 (2003), gr-qc/0211094.

[68] R. A. Breuer, ed., Gravitational perturbation theory and synchrotron radiation, vol. 44 of Lecture Notes in Physics, Berlin Springer Verlag (1975).

[69] S. Chandrasekhar, The mathematical theory of black holes (Oxford University Press, New York, 1992). 